\title{
Nomograms to predict survival after colorectal cancer resection without preoperative therapy
}

\author{
Zhen-yu Zhang' ${ }^{1}$ Qi-feng Luo', Xiao-wei Yin², Zhen-ling Dai', Shiva Basnet ${ }^{1}$ and Hai-yan Ge ${ }^{1 *}$
}

\begin{abstract}
Background: The predictive accuracy of the American Joint Committee on Cancer (AJCC) stages of colorectal cancer (CRC) is mediocre. This study aimed to develop postoperative nomograms to predict cancer-specific survival (CSS) and overall survival (OS) after CRC resection without preoperative therapy.

Methods: Eligible patients with stage I to IV CRC $(n=56072)$ diagnosed from 2004 to 2010 were selected from the Surveillance, Epidemiology, and End Results (SEER) database. The patients were allocated into training $(n=27,700)$, contemporary $(n=3158)$, and prospective $(n=25,214)$ validation cohorts. Clinically important variables were incorporated and selected using the Akaike information criterion in multivariate Cox regressions to derive nomograms with the training cohort. The performance of the nomograms was assessed and externally testified using the concordance index (c-index), bootstrap validation, calibration, time-dependent receiver-operating characteristic curves, Kaplan-Meier curves, mosaic plots, and decision curve analysis (DCA). Performance of the conventional AJCC stages was also compared with the nomograms using similar statistics.
\end{abstract}

Results: The nomograms for CSS and OS shared common predictors: sex, age, race, marital status, preoperative carcinoembryonic antigen status, surgical extent, tumor size, location, histology, differentiation, infiltration depth, lymph node count, lymph node ratio, and metastasis. The c-indexes of the nomograms for CSS and OS were 0.816 (95\% Cl 0.810-0.822) and 0.777 (95\% Cl 0.772-0.782), respectively. Performance evaluations showed that the nomograms achieved considerable predictive accuracy, appreciable reliability, and significant clinical validity with wide practical threshold probabilities, while the results remained reproducible when applied to the validation cohorts. Additionally, model comparisons and DCA proved that the nomograms excelled in stratifying each AJCC stage into three significant prognostic subgroups, allowing for more robust risk classification with an improved net benefit.

Conclusions: We propose two prognostic nomograms that exhibit improved predictive accuracy and net benefit for patients who have undergone CRC resection. The established nomograms are intended for risk assessment and selection of suitable patients who may benefit from adjuvant therapy and intensified follow-up after surgery. Independent external validations may still be required.

Keywords: Colorectal cancer, Nomogram, Cancer-specific survival, Overall survival, Decision curve analysis

Abbreviations: AIC, akaike information criterion; AJCC, American joint committee on cancer; AUC, area under the receiver-operating characteristic curve; CEA, carcinoembryonic antigen; $\mathrm{Cl}$, confident interval; $C R C$, colorectal cancer; CSS, cancer-specific survival; DCA, decision curve analysis; LNC, lymph node count; LNR, lymph node ratio; OS, overall survival; ROC, receiver-operating characteristic; SEER, surveillance, epidemiology, and end results

\footnotetext{
* Correspondence: gesurgery@163.com

'Department of Gastrointestinal Surgery, Shanghai East Hospital, Tongji

University School of Medicine, Pudong New District, No. 150, Jimo Road,

Shanghai 200120, China

Full list of author information is available at the end of the article
} 


\section{Background}

Colorectal cancer (CRC) is a leading contributor to cancer mortality worldwide $[1,2]$. Surgical treatment is the mainstay for elimination of CRC and continuity of life $[3,4]$. However, patients with a high risk of postoperative progression of CRC require additional interventions and informed decision-making with the help of physicians [3-5]. Among the vast spectrum of clinicopathological information $[3,6]$, the American Joint Committee on Cancer (AJCC) stages of CRC are fundamental for choosing optimal clinical interventions, and their use remains at the forefront of predicting and treating CRC [7]. Unfortunately, many observations are not consistent with the assumed relationship between advanced anatomical stages and reduced survival probabilities. For instance, disease recurs in $25 \%$ of patients with early CRC who are node-negative following curative resection [8]. Patients with stage II CRCs with low-risk features more frequently encounter adverse events than those with high-risk features [9]. Postoperative adjuvant therapies for patients with stage II CRC with fewer than 12 recovered nodes or other risk factors have not gained a clear survival benefit as expected [10-12]; however, a substantial improvement in survival has been achieved for patients with stage III CRC [11, 12]. Therapeutic effects only partially explain the conspicuous survival inhomogeneity within stage III CRC although stage migrations due to inadequate pathologic assessment may also play a role $[13,14]$. Metastatic CRC after curative hepatic resection has a 5-year overall survival (OS) of

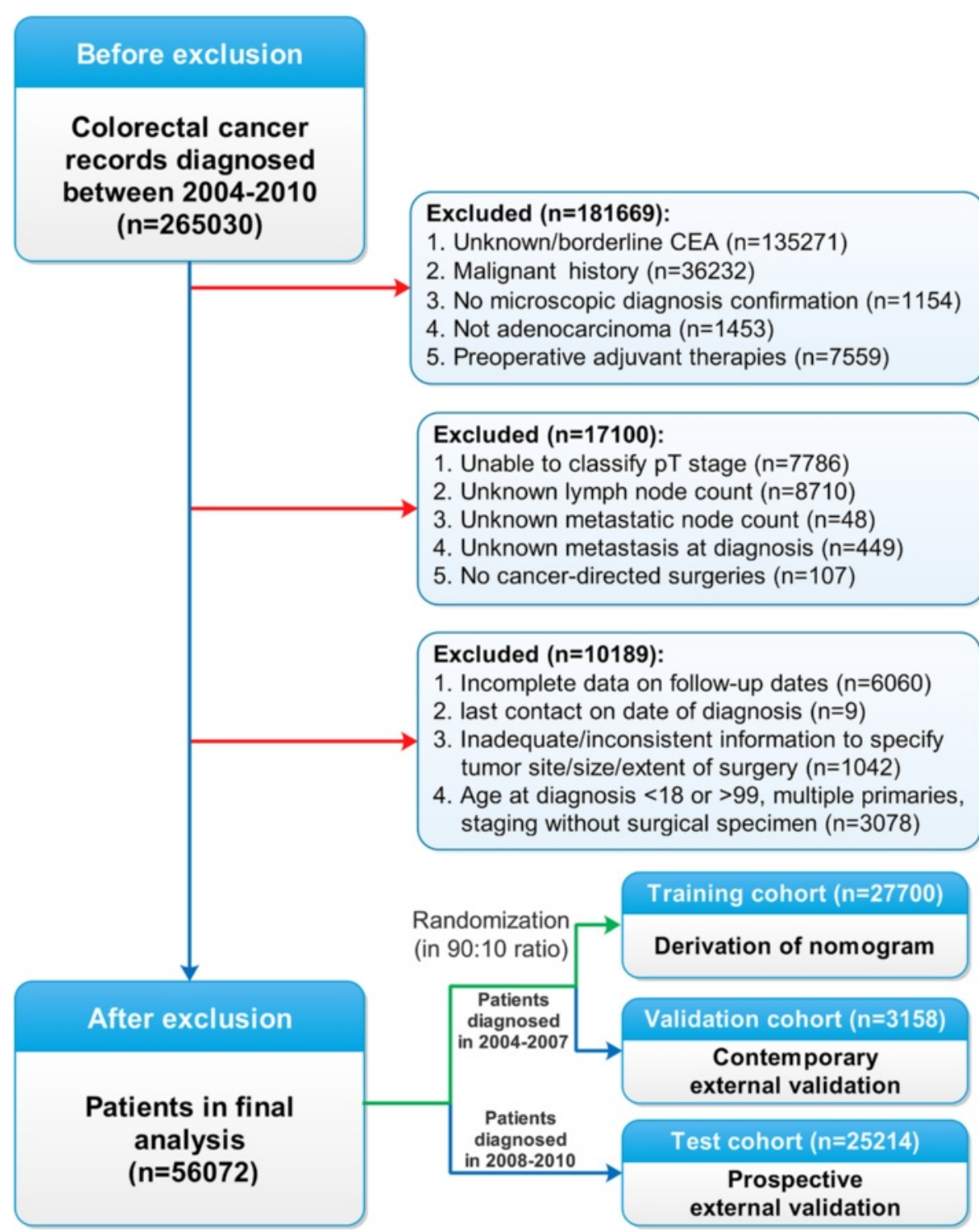

Fig. 1 Flow diagram of patient selection and study development 
Table 1 Characteristics of patients with colorectal cancer

\begin{tabular}{|c|c|c|c|c|c|c|}
\hline \multirow{2}{*}{$\begin{array}{l}\text { Variables } \\
\text { Sex, n, \% }\end{array}$} & \multicolumn{2}{|c|}{$\begin{array}{l}\text { Training cohort } \\
(n=27700)\end{array}$} & \multicolumn{2}{|c|}{$\begin{array}{l}\text { Validation cohort } \\
(n=3158)\end{array}$} & \multicolumn{2}{|c|}{$\begin{array}{l}\text { Test cohort } \\
(n=25214)\end{array}$} \\
\hline & & & & & & \\
\hline Female & 14077 & 50.8 & 1605 & 50.8 & 12702 & 50.4 \\
\hline Male & 13623 & 49.2 & 1553 & 49.2 & 12512 & 49.6 \\
\hline Age, year, median, range & 67 & $18-99$ & 67 & $18-99$ & 67 & $18-99$ \\
\hline \multicolumn{7}{|l|}{ Race, n, \% } \\
\hline White & 21722 & 78.4 & 2428 & 76.9 & 19710 & 78.2 \\
\hline Black & 3422 & 12.4 & 412 & 13.0 & 3106 & 12.3 \\
\hline Yellow (Chinese, Korean and Japanese) & 1229 & 4.4 & 169 & 5.4 & 1075 & 4.3 \\
\hline Other & 1327 & 4.8 & 149 & 4.7 & 1323 & 5.2 \\
\hline \multicolumn{7}{|l|}{ Marital status at diagnosis, n, \% } \\
\hline Married (including separated) & 15900 & 57.4 & 1812 & 57.3 & 14166 & 56.2 \\
\hline Divorced & 2378 & 8.6 & 266 & 8.4 & 2297 & 9.1 \\
\hline Single (never married) & 3519 & 12.7 & 406 & 12.9 & 3553 & 14.1 \\
\hline Widowed & 5185 & 18.7 & 601 & 19.1 & 4363 & 17.3 \\
\hline Unknown & 718 & 2.6 & 73 & 2.3 & 835 & 3.3 \\
\hline \multicolumn{7}{|l|}{ CEA status, $n, \%$} \\
\hline Negative & 15550 & 56.1 & 1803 & 57.1 & 14824 & 58.8 \\
\hline Positive & 12150 & 43.9 & 1355 & 42.9 & 10390 & 41.2 \\
\hline \multicolumn{7}{|l|}{ Tumor site, n, \% } \\
\hline Proximal colon (cecum to splenic flexure) & 14341 & 51.7 & 1621 & 51.3 & 13790 & 54.7 \\
\hline Distal colon (descending to sigmoid colon) & 8015 & 29.0 & 952 & 30.2 & 7441 & 29.5 \\
\hline Overlapping lesion of colon & 284 & 1.0 & 24 & 0.8 & 275 & 1.1 \\
\hline Rectum (including rectosigmoid junction) & 5060 & 18.3 & 561 & 17.7 & 3708 & 14.7 \\
\hline \multicolumn{7}{|l|}{ Tumor size, $n, \%$} \\
\hline$\leq 5 \mathrm{~cm}$ & 16861 & 60.9 & 1966 & 62.3 & 15178 & 60.2 \\
\hline$>5 \mathrm{~cm}$ & 9120 & 32.9 & 998 & 31.6 & 8557 & 33.9 \\
\hline Unknown & 1719 & 6.2 & 194 & 6.1 & 1479 & 5.9 \\
\hline \multicolumn{7}{|l|}{ Extent of surgery, $n, \%$} \\
\hline Local/segmental resection & 12879 & 46.5 & 1505 & 47.7 & 11464 & 45.5 \\
\hline Subtotal/hemisection & 13991 & 50.5 & 1549 & 49.0 & 13137 & 52.1 \\
\hline Total resection & 830 & 3.0 & 104 & 3.3 & 613 & 2.4 \\
\hline \multicolumn{7}{|l|}{ Histology, n, \% } \\
\hline Adenocarcinoma & 27375 & 98.8 & 3116 & 98.7 & 24975 & 99.1 \\
\hline Signet ring cell carcinoma & 325 & 1.2 & 42 & 1.3 & 239 & 0.9 \\
\hline \multicolumn{7}{|l|}{ Tumor grade, $n, \%$} \\
\hline Well to Moderately differentiated (G1 + G2) & 21137 & 76.3 & 2435 & 77.1 & 19450 & 77.2 \\
\hline Poorly to Undifferentiated (G3 + G4) & 5931 & 21.4 & 646 & 20.5 & 5202 & 20.6 \\
\hline Unknown & 632 & 2.3 & 77 & 2.4 & 562 & 2.2 \\
\hline \multicolumn{7}{|l|}{ pT stage, n, \% } \\
\hline pT1 & 2381 & 8.6 & 272 & 8.6 & 2662 & 10.6 \\
\hline pT2 & 3987 & 14.4 & 447 & 14.2 & 3844 & 15.2 \\
\hline рT3 & 17094 & 61.7 & 1980 & 62.7 & 14887 & 59.0 \\
\hline pT4a & 2244 & 8.1 & 256 & 8.1 & 2242 & 8.9 \\
\hline pT4b & 1994 & 7.2 & 203 & 6.4 & 1579 & 6.3 \\
\hline
\end{tabular}


Table 1 Characteristics of patients with colorectal cancer (Continued)

\begin{tabular}{|c|c|c|c|c|c|c|}
\hline \multicolumn{7}{|l|}{ pN stage, n, \% } \\
\hline NO & 14069 & 50.8 & 1615 & 51.1 & 13378 & 53.1 \\
\hline $\mathrm{N} 1 \mathrm{a}$ & 3445 & 12.4 & 402 & 12.7 & 3005 & 11.9 \\
\hline N1b & 3960 & 14.3 & 456 & 14.4 & 3378 & 13.4 \\
\hline $\mathrm{N} 2 \mathrm{a}$ & 3055 & 11.0 & 356 & 11.2 & 2611 & 10.4 \\
\hline $\mathrm{N} 2 \mathrm{~b}$ & 3171 & 11.5 & 329 & 10.4 & 2842 & 11.2 \\
\hline Lymph node count, mean, sd & 15.7 & 9.6 & 15.8 & 9.6 & 18.4 & 9.6 \\
\hline Lymph node ratio, mean, IQR & 0.16 & $0-0.24$ & 0.16 & $0-0.22$ & 0.13 & $0-0.18$ \\
\hline \multicolumn{7}{|l|}{ Metastasis, n, \% } \\
\hline MO & 22512 & 81.3 & 2587 & 81.9 & 21112 & 83.7 \\
\hline M1 & 5188 & 18.7 & 571 & 18.1 & 4102 & 16.3 \\
\hline Follow-up & 63 & $1-107$ & 64 & $1-107$ & 34 & $1-59$ \\
\hline Number of events & 9341 & 13359 & 1055 & 1496 & 5659 & 7689 \\
\hline 1-year cumulative survival & 87.9 & 84.1 & 88.6 & 84.7 & 89.8 & 86.5 \\
\hline 3-year cumulative survival & 73.8 & 67.1 & 75.1 & 68.7 & 77.3 & 70.9 \\
\hline 5-year cumulative survival ${ }^{a}$ & 66.6 & 57.2 & 67.7 & 58.6 & 70.8 & 60.6 \\
\hline
\end{tabular}

${ }^{a}$ Survival probabilities of the test cohort at 5 years were approximated at 59 months

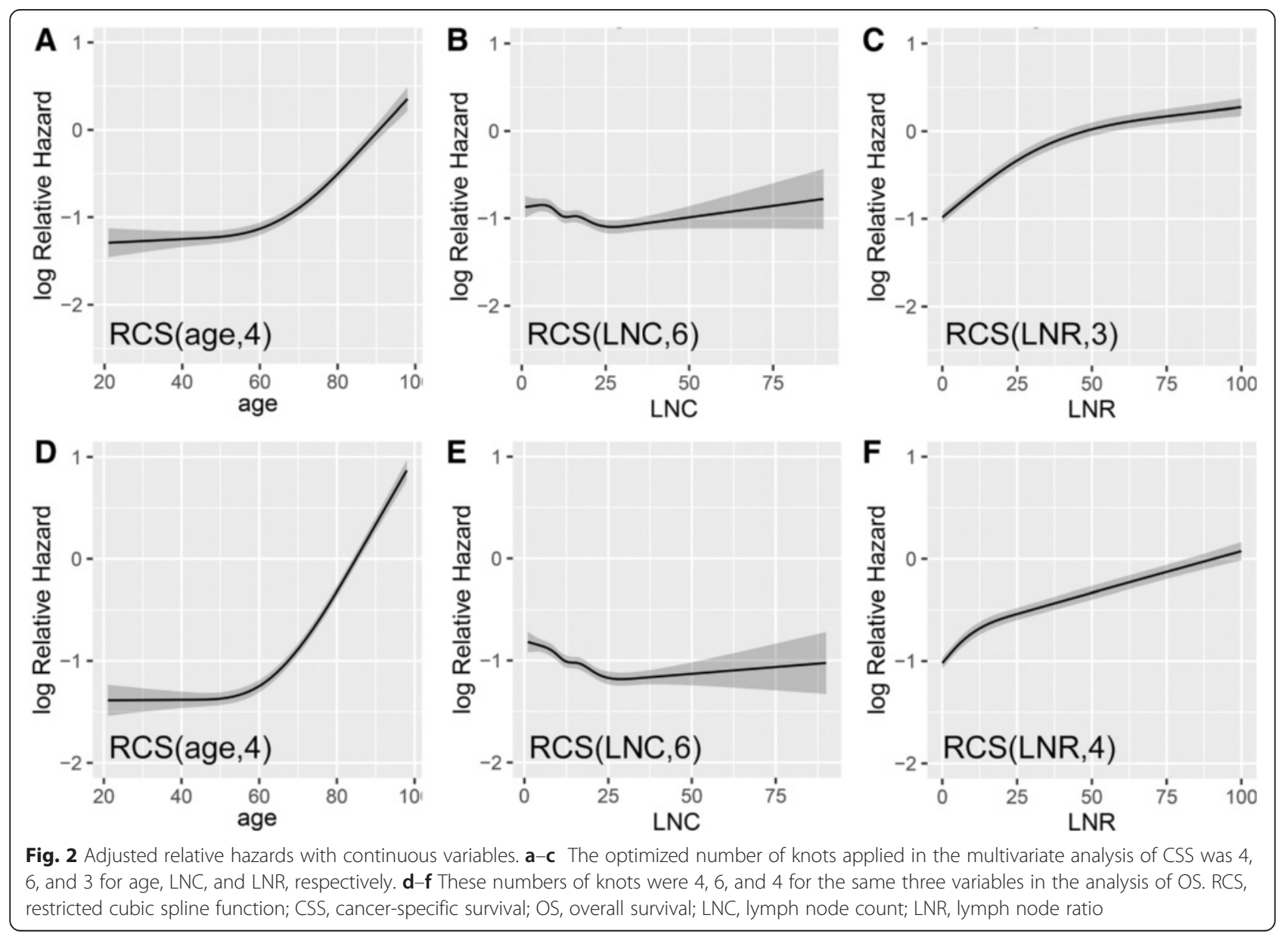


Table 2 Univariate cox regression analysis of training cohort

\begin{tabular}{|c|c|c|c|c|c|c|}
\hline \multirow[t]{2}{*}{ Variables } & \multicolumn{3}{|c|}{ Cancer-specific survival } & \multicolumn{3}{|c|}{ Overall survival } \\
\hline & $\mathrm{HR}$ & $95 \% \mathrm{Cl}$ & $P$ & $\mathrm{HR}$ & $95 \% \mathrm{Cl}$ & $P$ \\
\hline \multicolumn{7}{|l|}{ Sex } \\
\hline Female & ref & & & ref & & \\
\hline Male & 1.060 & $1.018-1.104$ & 0.0049 & 1.055 & $1.019-1.091$ & 0.0021 \\
\hline \multicolumn{7}{|l|}{ Race } \\
\hline White & ref & & & ref & & \\
\hline Black & 1.278 & $1.206-1.354$ & $<0.0001$ & 1.157 & $1.101-1.216$ & $<0.0001$ \\
\hline Yellow (Chinese, Korean and Japanese) & 0.762 & $0.682-0.850$ & $<0.0001$ & 0.701 & $0.638-0.770$ & $<0.0001$ \\
\hline Other & 0.974 & $0.884-1.072$ & 0.5850 & 0.870 & $0.800-0.946$ & 0.0011 \\
\hline \multicolumn{7}{|l|}{ Marital status at diagnosis } \\
\hline Married (including separated) & ref & & & ref & & \\
\hline Divorced & 1.171 & $1.088-1.259$ & $<0.0001$ & 1.175 & $1.103-1.251$ & $<0.0001$ \\
\hline Single (never married) & 1.332 & $1.255-1.414$ & $<0.0001$ & 1.316 & $1.250-1.386$ & $<0.0001$ \\
\hline Widowed & 1.376 & $1.305-1.450$ & $<0.0001$ & 1.746 & $1.675-1.821$ & $<0.0001$ \\
\hline Unknown & 1.045 & $0.914-1.196$ & 0.5175 & 1.246 & $1.120-1.387$ & 0.0001 \\
\hline \multicolumn{7}{|l|}{ CEA status } \\
\hline Negative & ref & & & ref & & \\
\hline Positive & 3.174 & $3.042-3.313$ & $<0.0001$ & 2.449 & $2.366-2.535$ & $<0.0001$ \\
\hline \multicolumn{7}{|l|}{ Tumor site } \\
\hline Proximal colon & ref & & & ref & & \\
\hline Distal colon & 0.892 & $0.850-0.935$ & $<0.0001$ & 0.835 & $0.803-0.869$ & $<0.0001$ \\
\hline Overlapping lesion of colon & 1.382 & $1.153-1.657$ & 0.0005 & 1.294 & $1.108-1.510$ & 0.0011 \\
\hline Rectum & 0.880 & $0.833-0.931$ & $<0.0001$ & 0.825 & $0.787-0.865$ & $<0.0001$ \\
\hline \multicolumn{7}{|l|}{ Tumor size } \\
\hline$\leq 5 \mathrm{~cm}$ & ref & & & ref & & \\
\hline$>5 \mathrm{~cm}$ & 1.429 & $1.370-1.491$ & $<0.0001$ & 1.316 & $1.270-1.363$ & $<0.0001$ \\
\hline Unknown & 0.821 & $0.746-0.904$ & 0.0001 & 0.806 & $0.745-0.873$ & $<0.0001$ \\
\hline \multicolumn{7}{|l|}{ Extent of surgery } \\
\hline Local/segmental resection & ref & & & ref & & \\
\hline Subtotal/hemisection & 1.161 & $1.114-1.211$ & $<0.0001$ & 1.181 & $1.141-1.223$ & $<0.0001$ \\
\hline Total resection & 1.527 & $1.373-1.699$ & $<0.0001$ & 1.388 & $1.264-1.525$ & $<0.0001$ \\
\hline \multicolumn{7}{|l|}{ Histology } \\
\hline Adenocarcinoma & ref & & & ref & & \\
\hline Signet ring cell carcinoma & 2.648 & $2.304-3.044$ & $<0.0001$ & 2.180 & $1.916-2.480$ & $<0.0001$ \\
\hline \multicolumn{7}{|l|}{ Tumor grade } \\
\hline G1/G2 & ref & & & ref & & \\
\hline G3/G4 & 1.903 & $1.820-1.989$ & $<0.0001$ & 1.614 & $1.553-1.678$ & $<0.0001$ \\
\hline Unknown & 1.007 & $0.871-1.164$ & 0.9283 & 0.942 & $0.834-1.064$ & 0.3372 \\
\hline \multicolumn{7}{|l|}{ pT stage } \\
\hline pT1 & ref & & & ref & & \\
\hline pT2 & 1.854 & $1.556-2.209$ & $<0.0001$ & 1.662 & $1.495-1.848$ & $<0.0001$ \\
\hline pT3 & 6.161 & $5.285-7.182$ & $<0.0001$ & 3.150 & $2.871-1.848$ & $<0.0001$ \\
\hline pT4a & 13.422 & $11.43-15.760$ & $<0.0001$ & 5.775 & $5.21-6.400$ & $<0.0001$ \\
\hline pT4b & 17.429 & $14.844-20.464$ & $<0.0001$ & 7.324 & $6.607-8.120$ & $<0.0001$ \\
\hline
\end{tabular}


Table 2 Univariate cox regression analysis of training cohort (Continued)

\begin{tabular}{|c|c|c|c|c|c|c|}
\hline \multicolumn{7}{|c|}{ Metastasis } \\
\hline MO & ref & & & ref & & \\
\hline M1 & 7.733 & $7.414-8.066$ & $<0.0001$ & 4.953 & $4.773-5.139$ & $<0.0001$ \\
\hline
\end{tabular}

HR hazard ratio, $95 \% \mathrm{Cl} 95 \%$ confident interval, ref reference category, CEA carcinoembryonic antigen

Table 3 Multivariate cox regression analysis of training cohort

\begin{tabular}{|c|c|c|c|c|c|c|}
\hline \multirow[b]{2}{*}{ Covariates } & \multicolumn{3}{|c|}{ Cancer-specific survival } & \multicolumn{3}{|c|}{ Overall survival } \\
\hline & $\mathrm{HR}$ & $95 \% \mathrm{Cl}$ & $P$ & $\mathrm{HR}$ & $95 \% \mathrm{Cl}$ & $P$ \\
\hline Sex (Male vs. Female) & 1.142 & $1.094-1.193$ & $<0.0001$ & 1.246 & $1.202-1.293$ & $<0.0001$ \\
\hline \multicolumn{7}{|l|}{ Race (ref, White) } \\
\hline Black & 1.199 & $1.130-1.273$ & $<0.0001$ & 1.191 & $1.132-1.254$ & $<0.0001$ \\
\hline Yellow (Chinese, Korean, Japanese) & 0.784 & $0.702-0.875$ & $<0.0001$ & 0.713 & $0.649-0.784$ & $<0.0001$ \\
\hline Other & 1.030 & $0.935-1.135$ & 0.5449 & 0.996 & $0.915-1.083$ & 0.9203 \\
\hline \multicolumn{7}{|l|}{ Marital status at diagnosis (ref, Married) } \\
\hline Divorced & 1.175 & $1.092-1.265$ & $<0.0001$ & 1.214 & $1.139-1.293$ & $<0.0001$ \\
\hline Single (never married) & 1.235 & $1.161-1.313$ & $<0.0001$ & 1.277 & $1.211-1.346$ & $<0.0001$ \\
\hline Widowed & 1.189 & $1.118-1.264$ & $<0.0001$ & 1.224 & $1.165-1.285$ & $<0.0001$ \\
\hline Unknown & 1.014 & $0.886-1.161$ & 0.8424 & 1.145 & $1.029-1.275$ & 0.0131 \\
\hline CEA status (Positive vs. negative) & 1.589 & $1.517-1.664$ & $<0.0001$ & 1.486 & $1.431-1.543$ & $<0.0001$ \\
\hline \multicolumn{7}{|l|}{ Extent of surgery (ref, Loc/seg resection) } \\
\hline Subtotal/hemisection & 1.067 & $1.012-1.125$ & 0.0156 & 1.040 & $0.995-1.087$ & 0.0824 \\
\hline Total resection & 1.269 & $1.139-1.414$ & $<0.0001$ & 1.180 & $1.073-1.297$ & 0.0007 \\
\hline \multicolumn{7}{|l|}{ Tumor site (ref, Proximal colon) } \\
\hline Distal colon & 0.910 & $0.860-0.962$ & $<0.0001$ & 0.918 & $0.876-0.962$ & 0.0004 \\
\hline Overlapping lesion of colon & 1.090 & $0.909-1.307$ & 0.3545 & 1.111 & $0.952-1.298$ & 0.1824 \\
\hline Rectum & 1.002 & $0.935-1.074$ & 0.9516 & 0.981 & $0.926-1.040$ & 0.5211 \\
\hline \multicolumn{7}{|l|}{ Tumor size (ref, $\leq 5 \mathrm{~cm}$ ) } \\
\hline$>5 \mathrm{~cm}$ & 1.029 & $0.984-1.076$ & 0.2050 & 1.026 & $0.989-1.066$ & 0.1741 \\
\hline Unknown & 1.141 & $1.035-1.258$ & 0.0078 & 1.075 & $0.991-1.166$ & 0.0810 \\
\hline \multicolumn{7}{|l|}{ Histology (ref, Adenocarcinoma) } \\
\hline Signet ring cell carcinoma & 1.409 & $1.220-1.626$ & $<0.0001$ & 1.380 & $1.209-1.575$ & $<0.0001$ \\
\hline \multicolumn{7}{|l|}{ Tumor grade (ref, G1/G2) } \\
\hline G3/G4 & 1.278 & $1.219-1.339$ & $<0.0001$ & 1.184 & $1.137-1.233$ & $<0.0001$ \\
\hline Unknown & 1.143 & $0.987-1.323$ & 0.0736 & 1.049 & $0.927-1.186$ & 0.4501 \\
\hline \multicolumn{7}{|l|}{ pT stage (ref, pT1) } \\
\hline pT2 & 1.567 & $1.312-1.872$ & $<0.0001$ & 1.398 & $1.254-1.558$ & $<0.0001$ \\
\hline pT3 & 2.949 & $2.516-3.457$ & $<0.0001$ & 1.796 & $1.628-1.981$ & $<0.0001$ \\
\hline pT4a & 4.429 & $3.746-5.237$ & $<0.0001$ & 2.508 & $2.247-2.799$ & $<0.0001$ \\
\hline pT4b & 4.760 & $4.021-5.634$ & $<0.0001$ & 2.746 & $2.456-3.069$ & $<0.0001$ \\
\hline Metastasis (M1 vs. M0) & 4.075 & $3.876-4.284$ & $<0.0001$ & 3.357 & $3.213-3.508$ & $<0.0001$ \\
\hline
\end{tabular}




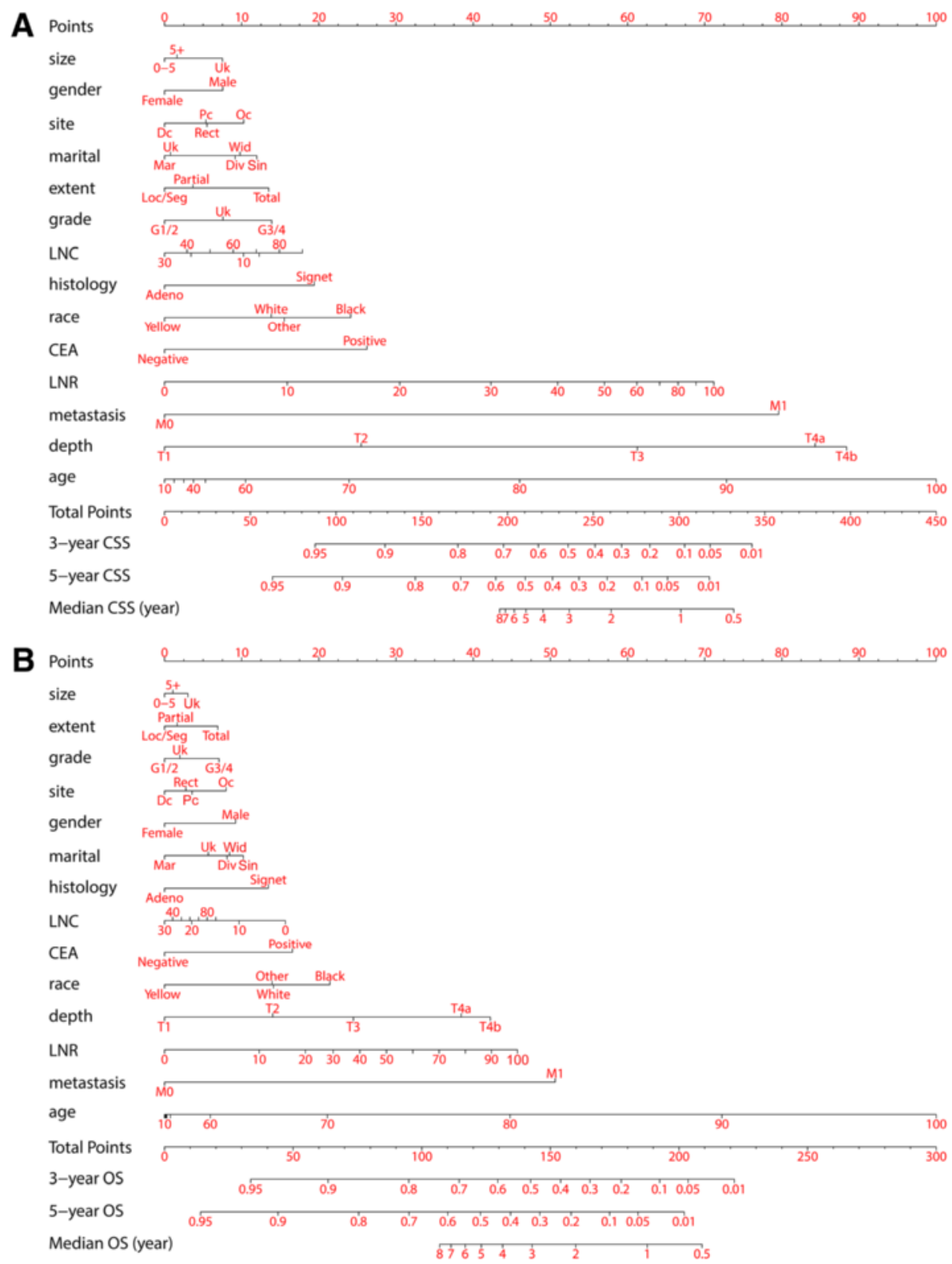

Fig. 3 Nomograms for (a) CSS and (b) OS. Generally, each individual involved covariate was assessed for the patient and given a point on the basis of the nomograms. Aggregated points were obtained by summing the given points of all involved variables. The aggregated points corresponded to particular survival probabilities and median survival years that could be indicated by the nomograms. A higher total number of points frequently indicated a higher possibility of adverse outcomes (CSS or OS) and therefore a lower expected survival. CSS, cancer-specific survival; OS, overall survival

47.7 to $57.6 \%[15,16]$, while the OS of most patients with unresectable metastatic CRC is extremely poor [17]. Survival of CRC remains poor for multiple reasons that are not limited to tumor-related factors. Despite the increased complexity among several modifications of the AJCC cancer staging manuals [14], the AJCC stages have intrinsic defects as an anatomy-dependent rather than multidiscipline-integrated metric [18]. Moreover, the AJCC stages force categorization of tumor dissemination in a stepwise fashion, causing additional loss of predictive accuracy $[18,19]$. A consequential issue has thus emerged: both the 5-year cancer-specific survival (CSS) and OS are heterogeneous among patients with the same stage of CRC [14].

Many useful factors are not sufficiently utilized in clinical prognostication. The plasma carcinoembryonic antigen (CEA) concentration is strongly predictive $[15,19]$ and plays roles in staging other than indicating recurrence. Patient-specific factors such as sex, ethnicity, and marital status are also associated with 
Table 4 Points for categorical variables in nomograms

\begin{tabular}{|c|c|c|c|}
\hline \multirow{2}{*}{ Variables } & \multirow[b]{2}{*}{ Labels for tick marks } & \multicolumn{2}{|c|}{ Points } \\
\hline & & CSS & OS \\
\hline \multicolumn{4}{|l|}{ Sex } \\
\hline Female & Female & 0 & 0 \\
\hline Male & Male & 7.5 & 9.2 \\
\hline \multicolumn{4}{|l|}{ Race } \\
\hline White & White & 13.8 & 14.1 \\
\hline Black & Black & 24.1 & 21.4 \\
\hline Yellow (Chinese, Korean and Japanese) & Yellow & 0 & 0 \\
\hline Other & Other & 15.5 & 13.9 \\
\hline \multicolumn{4}{|l|}{ Marital status at diagnosis } \\
\hline Married (including separated) & Mar & 0 & 0 \\
\hline Divorced & Div & 9.2 & 8.1 \\
\hline Single (never married) & $\operatorname{Sin}$ & 11.9 & 10.2 \\
\hline Widowed & Wid & 9.8 & 8.4 \\
\hline Unknown & Uk & 0.8 & 5.7 \\
\hline \multicolumn{4}{|l|}{ CEA status } \\
\hline Negative & Negative & 0 & 0 \\
\hline Positive & Positive & 26.2 & 16.6 \\
\hline \multicolumn{4}{|l|}{ Tumor site } \\
\hline Proximal colon (cecum to splenic flexure) & Pc & 5.4 & 3.6 \\
\hline Distal colon (descending to sigmoid colon) & Dc & 0 & 0 \\
\hline Overlapping lesion of colon & Oc & 10.3 & 8.0 \\
\hline Rectum (including rectosigmoid junction) & Rect & 5.5 & 2.8 \\
\hline \multicolumn{4}{|l|}{ Tumor size } \\
\hline$\leq 5 \mathrm{~cm}$ & $0-5$ & 0 & 0 \\
\hline$>5 \mathrm{~cm}$ & $5+$ & 1.6 & 1.1 \\
\hline Unknown & Uk & 7.5 & 3.0 \\
\hline \multicolumn{4}{|l|}{ Extent of surgery } \\
\hline Local/segmental resection & Loc/Seg & 0 & 0 \\
\hline Subtotal/hemisection & Partial & 3.7 & 1.6 \\
\hline Total resection & Total & 13.5 & 6.9 \\
\hline \multicolumn{4}{|l|}{ Histology } \\
\hline Adenocarcinoma & Adeno & 0 & 0 \\
\hline Signet ring cell carcinoma & Signet & 19.4 & 13.5 \\
\hline \multicolumn{4}{|l|}{ Tumor grade } \\
\hline Well to Moderately differentiated (G1 + G2) & $\mathrm{G} 1 / 2$ & 0 & 0 \\
\hline Poorly to Undifferentiated (G3 + G4) & $\mathrm{G} 3 / 4$ & 13.9 & 7.1 \\
\hline Unknown & Uk & 7.6 & 2.0 \\
\hline \multicolumn{4}{|l|}{ pT stage } \\
\hline pT1 & $\mathrm{T} 1$ & 0 & 0 \\
\hline pT2 & T2 & 25.5 & 14.0 \\
\hline pT3 & T3 & 61.3 & 24.5 \\
\hline pT4a & T4a & 84.3 & 38.4 \\
\hline pT4b & T4b & 88.4 & 42.2 \\
\hline
\end{tabular}


Table 4 Points for categorical variables in nomograms (Continued)

\begin{tabular}{llll}
\hline Metastasis & & & \\
M0 & M0 & 0 & 0 \\
M1 & M1 & 79.6 & 50.6 \\
\hline
\end{tabular}

CSS cancer-specific survival, OS overall survival, CEA carcinoembryonic antigen

survival $[1,2,20,21]$, representing untapped information that may be useful for individualized therapies and outcomes. Many other parameters included in routine pathologic reports are also apparent survival determinants, including tumor location, size, histology, grade, differentiation, lymph node count (LNC), lymph node ratio (LNR), and surgical extent [6]. All of these elements are inseparable qualities of a "successful cancer career," of which more detailed evaluations are still required, however.

We anticipate that the combined performance of the above-mentioned factors is superior to that of the AJCC stages and may serve as a more precise and reproducible tool for individualized survival estimations. We have herein incorporated clinically important variables with data from the Surveillance, Epidemiology, and End Results (SEER) database to develop validated prognostic nomograms for predicting CSS and OS of patients with surgically treated CRC without neoadjuvant therapies.

\section{Methods}

\section{Patient eligibility and variables}

The SEER program is a national database and primary source of cancer statistics that is currently maintained by the National Cancer Institute [22]. The data of patients with CRC diagnosed from 2004 to 2010 were retrieved from the SEER research database using the SEER*Stat program ( $v$ 8.2.1) [22]. In total, 265,030 records were retrieved. Any surgically treated, pathologically proven, staged colorectal adenocarcinomas were considered. Only patients who met the following criteria were included in the formal analysis: (1) known preoperative CEA status, (2) no history of malignancy, (3) microscopically proven stage I to IV primary adenocarcinoma (including signet ring cell carcinoma), (3) no adjuvant therapy before surgery, (4) cancer-directed surgery of primary tumors with sufficient information to specify the $\mathrm{T} / \mathrm{N} / \mathrm{M}$ stage and LNC/LNR, (5) active follow-up with complete date and known outcome, and (6) adequate/consistent information to specify the primary tumor site, size, and other variables. Patients aged $<18$ or $>99$ years and those with multiple primary cancers were excluded. Patients were also excluded if their $\mathrm{T} / \mathrm{N}$ did not meet pathological staging criteria $($ not $\mathrm{pT} / \mathrm{N})$. These patients were small in number, but they might have introduced bias to the survival analysis; thus, they were excluded.

The following variables were assessed: sex, age, race, marital status, year of diagnosis, primary tumor location, size, histology, grade, TNM stage, LNC, cause-specific death, and vital status. Cancer stages reported using the 6th AJCC/TNM stages (AJCC6) were converted based on the 7th edition (AJCC7). The LNR was calculated by dividing metastatic node number by the LNC.

After patient exclusion based on the above-mentioned criteria, 56,072 eligible patients were identified. Patients diagnosed from 2004 to 2007 were randomized into a training cohort and a contemporary validation cohort (ratio, 90:10).

Table 5 Points for continuous variables in nomograms

\begin{tabular}{|c|c|c|c|c|c|c|c|c|}
\hline \multicolumn{3}{|c|}{ Age at diagnosis } & \multicolumn{3}{|c|}{ Lymph node count, $n$} & \multicolumn{3}{|c|}{$\underline{\text { Lymph node ratio }}$} \\
\hline Values, no. & Pts for CSS & Pts for OS & Values, no. & Pts for CSS & Pts for OS & Values, \% & Pts for CSS & Pts for OS \\
\hline 10 & 0.0 & 0.0 & 0 & 12.3 & 15.7 & 0 & 0.0 & 0.0 \\
\hline 20 & 1.2 & 0.1 & 10 & 10.2 & 9.7 & 10 & 15.9 & 12.3 \\
\hline 30 & 2.5 & 0.2 & 20 & 3.4 & 3.5 & 20 & 30.5 & 18.2 \\
\hline 40 & 3.7 & 0.3 & 30 & 0.0 & 0.0 & 30 & 42.3 & 21.8 \\
\hline 50 & 5.3 & 0.8 & 40 & 2.9 & 1.1 & 40 & 50.9 & 25.3 \\
\hline 60 & 10.5 & 5.9 & 50 & 5.9 & 2.2 & 50 & 57.0 & 28.8 \\
\hline 70 & 23.9 & 21.1 & 60 & 8.9 & 3.3 & 60 & 61.2 & 32.2 \\
\hline 80 & 46.0 & 44.8 & 70 & 11.9 & 4.4 & 70 & 64.2 & 35.6 \\
\hline 90 & 72.8 & 72.2 & 80 & 14.9 & 5.5 & 80 & 66.5 & 39.0 \\
\hline \multirow[t]{2}{*}{100} & 100.0 & 100.0 & 90 & 17.9 & 6.6 & 90 & 68.9 & 42.4 \\
\hline & & & & & & 100 & 71.2 & 45.7 \\
\hline
\end{tabular}

Pts points, CSS cancer-specific survival, OS overall survival 
The remaining patients were diagnosed from 2008 to 2010 and were thus assigned to a prospective test cohort (Fig. 1).

\section{Statistical methods}

Discontinuous variables were categorized before modeling based on clinical reasoning and significance. Linear assumptions of continuous variables (age, LNC, and LNR) were relaxed with restricted cubic spline functions to determine the optimal number of knots by maximizing goodness of fit using the log-likelihood and minimizing information loss using the Akaike information criterion (AIC) [23]. Multivariate models for nomograms were built by incorporating significant variables from univariate Cox proportional hazard regressions in a backward stepwise manner based on the AIC. Model performance was appraised using the concordance index (c-index) and internally testified by 200 -sample bootstrap validation and calibration. External validation was performed by applying nomograms to the contemporary validation cohort and prospective test cohort separately, followed by evaluation of similar statistics in the new data sets. Different c-indexes were compared using the compareC [24] package. Next, patients in all cohorts were given an aggregated score using standard points derived from the nomograms. Time-dependent receiveroperating characteristic (ROC) curve analysis was performed with the timeROC [25] package to evaluate the performance of the nomograms with the accumulated scores as a continuous predictive variable. The nomograms were compared with the AJCC6/7 stages by risk classification and stratification using Kaplan-Meier survival curves and statistically clarified by quantifying the cumulative 5-year survival and hazard ratios for each stratum. Briefly, risk classification was achieved by ranking the accumulated nomogram scores by deciles to derive 10 risk groups (Nomo stages) with patients in the training cohort. For risk stratification, the patients were divided by score tertiles for each AJCC7 substage to generate three prognostic strata: low-, median-, and high-risk. The two external cohorts were likewise classified and stratified according to thresholds defined by the training cohort. Next, mosaic plots were drawn to demonstrate the AJCC7 stage distributions in contrast with the Nomo stages. After addressing the accuracy of the nomograms, decision curve analysis (DCA) [26] was performed to finalize the ranges
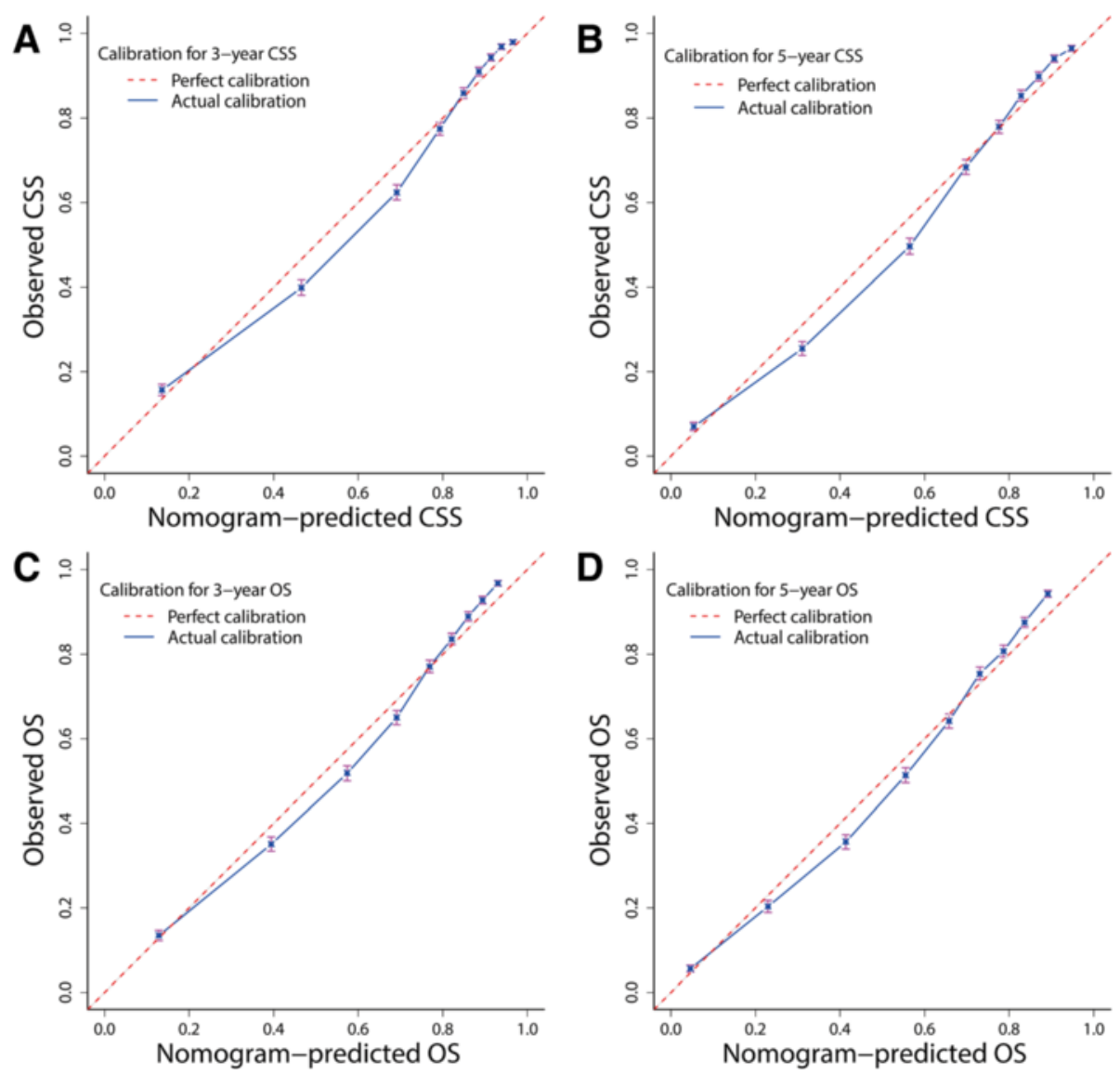

Fig. 4 Bootstrap calibrations of nomograms. The predicted 3- and 5-year ( $\mathbf{a}, \mathbf{b})$ CSS and (c, d) OS were well correlated with the actual survival probabilities 
of threshold probabilities within which the nomograms were clinically valuable. The patients were randomly allocated using the PASW 18.0 program (SPSS Inc., Chicago, IL); the other analyses were processed with the $\mathrm{R}$ program ( $\mathrm{v}$ 3.2.3) using rms [23] and the above-mentioned packages. Only a two-tailed $P$ value of $<0.05$ was considered statistically significant. This study followed the TRIPOD statement [27] and adhered to the Declaration of Helsinki for medical research involving human subjects [28].

\section{Results}

Baseline characteristics

The characteristics of the patients in the derivation and validation cohorts are shown in Table 1.
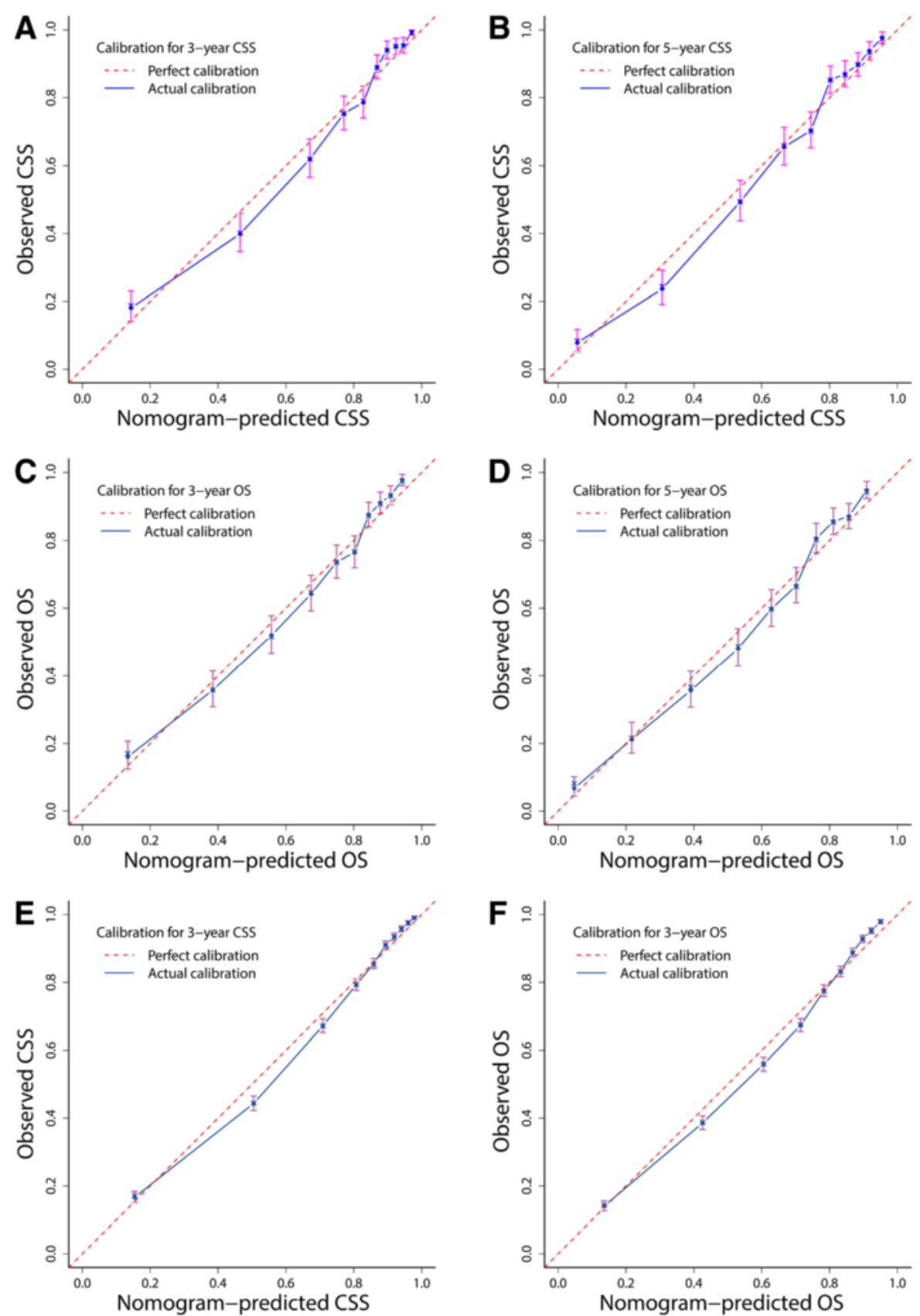

Fig. 5 Bootstrap calibration of nomograms in the external cohorts. The nomograms were externally calibrated in the validation cohort by predicting the 3- and 5-year (a, b) CSS and (c, d) OS. The nomograms were also calibrated in the test cohort for the 3-year (e) CSS and (f) OS. All results showed good validation. CSS, cancer-specific survival; OS, overall survival 
Table 6 Time-dependent receiver-operating characteristic curves analysis

\begin{tabular}{|c|c|c|c|c|c|c|c|c|}
\hline \multirow[b]{3}{*}{ Study cohort } & \multicolumn{4}{|c|}{ Cancer-specific survival } & \multicolumn{4}{|c|}{ Overall survival } \\
\hline & \multicolumn{4}{|c|}{ AUC, $\%$} & \multicolumn{4}{|l|}{ AUC, $\%$} \\
\hline & 1 year & 3 years & 5 years & 7 years & 1 year & 3 years & 5 years & 7 years \\
\hline Training cohort & 85.2 & 87.6 & 87.5 & 86.6 & 82.1 & 84.1 & 84.2 & 83.6 \\
\hline Validation cohort & 83.0 & 87.1 & 86.7 & 85.5 & 80.6 & 83.7 & 83.7 & 82.7 \\
\hline Test cohort & 86.0 & 87.8 & / & / & 83.1 & 84.6 & / & / \\
\hline
\end{tabular}

$A \cup C$ area under the time-dependent receiver-operating characteristic curves

\section{Cox regression of training cohort}

No continuous variables (age, LNC, or LNR) had linear effects on either CSS or OS (Fig. 2). All variables assessed in the univariate analysis (Table 2) remained significant in the multivariate Cox regressions except tumor size (Table 3).

\section{Nomograms for CSS and OS}

As selected by the AIC, all tested covariates were employed in the nomograms. The c-indexes were 0.816
(95\% CI 0.810-0.822) and 0.777 (95\% CI 0.772-0.782) for the CSS (Fig. 3a) and OS (Fig. 3b) predictive nomograms, respectively. Details of the nomograms' labels and points were shown in Table 4 and Table 5.

\section{Internal validation}

The bootstrap-corrected c-indexes (CSS, 0.8157; OS, 0.7768) were close to those of the nomograms. Both models exhibited good validation.

Table 7 Comparison of nomogram with AJCC staging system

\begin{tabular}{|c|c|c|c|c|}
\hline & Nomogram score & $7^{\text {th }}$ AJCC stage & $6^{\text {th }}$ AJCC stage & $P$ \\
\hline \multicolumn{5}{|l|}{ Training cohort, CSS } \\
\hline AIC & 172262 & 174703 & 174949 & / \\
\hline Log-likelihood & -86130 & -87344 & -87468 & All $<0.0001$ \\
\hline C-index (95 \% Cl) & $0.816(0.810-0.822)$ & $0.777(0.771-0.783)$ & $0.774(0.768-0.780)$ & All $<0.0001$ \\
\hline \multicolumn{5}{|l|}{ Training cohort, OS } \\
\hline AIC & 250348 & 255973 & 256182 & / \\
\hline Log-likelihood & -125173 & -127979 & -128085 & All $<0.0001$ \\
\hline C-index (95 \% Cl) & $0.777(0.772-0.782)$ & $0.698(0.693-0.0 .703)$ & $0.696(0.691-0.701)$ & All $<0.0001$ \\
\hline \multicolumn{5}{|l|}{ Validation cohort, CSS } \\
\hline AIC & 14983 & 15261 & 15272 & / \\
\hline Log-likelihood $^{a}$ & -7490 & -7623 & -7630 & All $<0.0001$ \\
\hline C-index ${ }^{\mathrm{b}}(95 \%$ Cl) & $0.809(0.791-0.827)$ & $0.770(0.752-0.788)$ & $0.768(0.750-0.786)$ & All $<0.0001$ \\
\hline \multicolumn{5}{|l|}{ Validation cohort, OS } \\
\hline AIC & 21611 & 22235 & 22244 & / \\
\hline Log-likelihood $^{c}$ & -10805 & -11110 & -11116 & All $<0.0001$ \\
\hline C-index ${ }^{d}(95 \%$ Cl) & $0.773(0.757-0.789)$ & $0.699(0.683-0.715)$ & $0.697(0.681-0.713)$ & All $<0.0001$ \\
\hline \multicolumn{5}{|l|}{ Test cohort, CSS } \\
\hline AlC & 102103 & 104057 & 105039 & / \\
\hline Log-likelihood & -51050 & -52021 & -52519 & All $<0.0001$ \\
\hline C-index (95 \% Cl) & $0.838(0.830-0.846)$ & $0.794(0.786-0.802)$ & $0.786(0.778-0.794)$ & All $<0.0001$ \\
\hline \multicolumn{5}{|l|}{ Test cohort, OS } \\
\hline AIC & 141606 & 145343 & 146456 & / \\
\hline Log-likelihood & -70802 & -72664 & -73227 & All $<0.0001$ \\
\hline C-index (95 \% Cl) & $0.802(0.796-0.808)$ & $0.723(0.717-0.729)$ & $0.715(0.709-0.721)$ & All $<0.0001$ \\
\hline
\end{tabular}

AJCC American joint committee on cancer, AIC akaike information criterion, C-index concordance index, $95 \% \mathrm{Cl} 95 \%$ confident interval ${ }^{\text {a }}$ The $P$ value comparing $6^{\text {th }}$ and $7^{\text {th }}$ AJCC stage was 0.0003

${ }^{\mathrm{b}}$ The $P$ value comparing $6^{\text {th }}$ and $7^{\text {th }}$ AJCC stage was 0.5187

'The $P$ value comparing $6^{\text {th }}$ and $7^{\text {th }}$ AJCC stage was 0.0006

${ }^{\mathrm{d}}$ The $P$ value comparing $6^{\text {th }}$ and $7^{\text {th }}$ AJCC stage was 0.3709 


\section{Nomogram calibration}

As shown in the calibration plots, the nomogrampredicted 3- and 5-year CSS and OS were well correlated with the corresponding Kaplan-Meier estimates (Fig. 4), suggesting appreciable reliability of the nomograms.

\section{External validation}

The c-indexes of the nomograms for prediction of CSS and OS were 0.809 (95\% CI 0.791-0.827) and 0.773 (95\% CI 0.757-0.789) in the validation cohort, while the optimism-corrected c-indexes were 0.804 and 0.768 , respectively. In the test cohort, the c-indexes were 0.839 (95\% CI 0.830-0.846) and 0.802 (95\% CI 0.796-0.808) with corrected estimates of 0.838 and 0.801 for CSS and
OS prediction, respectively. The external calibration plots also showed good validation (Fig. 5).

\section{Time-dependent ROC curve analysis}

The areas under the ROC curve (AUCs) for predicting CSS ranged from 83.0 to $87.8 \%$ in the three cohorts from 1 to 7 years. The AUCs for predicting OS varied from 80.6 to $84.6 \%$ during the same years (Table 6). The nomograms exhibited considerable efficiency to discriminate outcomes.

\section{Comparison of nomograms with AJCC stages}

First, when compared with the AJCC6/7 stages, the nomograms yielded the largest log-likelihoods and c-indexes

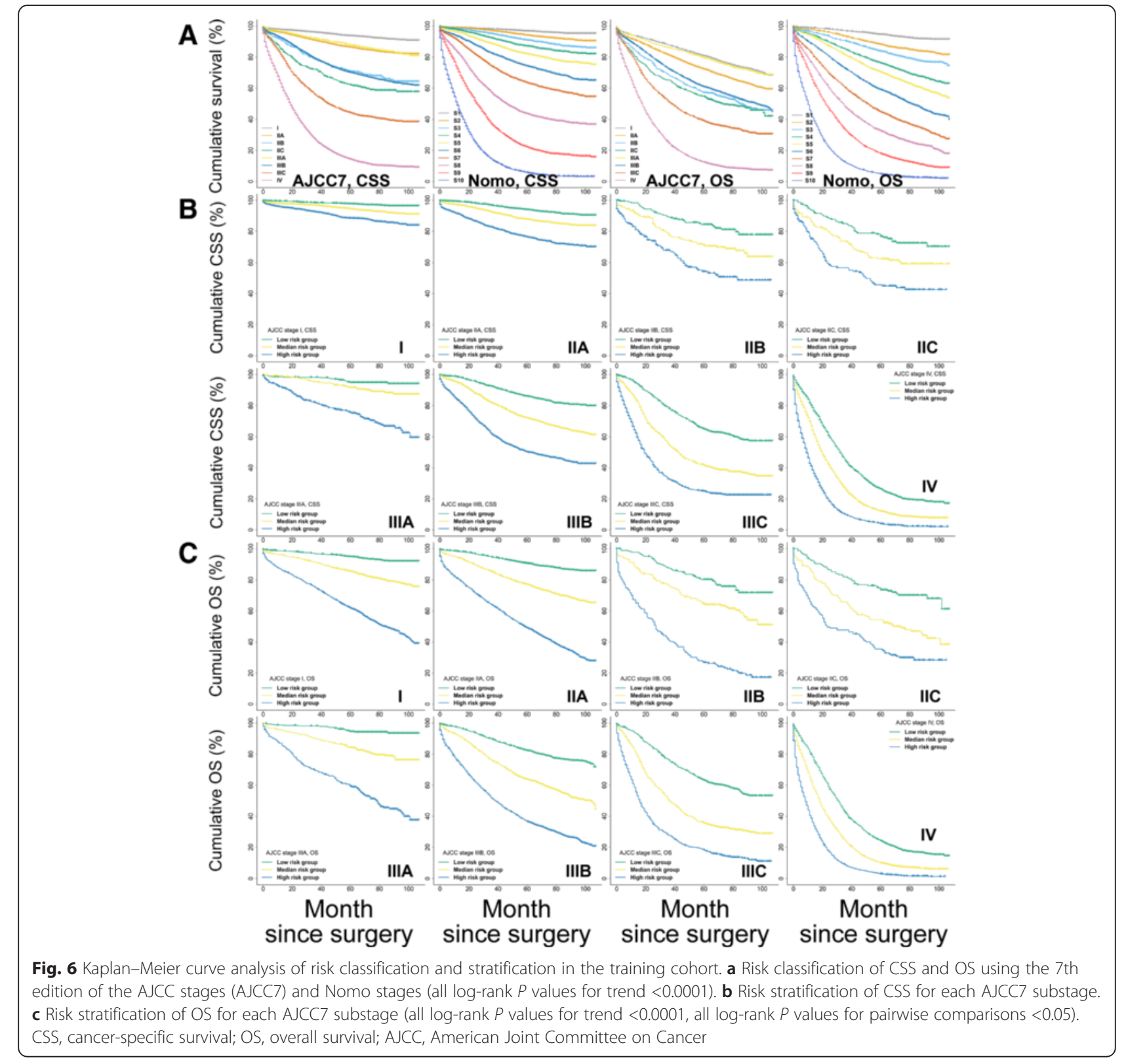


along with the smallest AIC values for CSS and OS prediction in all cohorts (Table 7). These results imply that the nomograms were more robust than the AJCC stages.

Second, as depicted by the Kaplan-Meier curves, the ability of the AJCC7 stages to discriminate CSS and OS was mediocre in both the training (Fig. 6a) and external cohorts (Fig. 7). However, the Nomo stages performed consistently much better in all cohorts (Figs. 6a and 7). Further analysis in the training (Fig. $6 \mathrm{~b}$ and c) and test cohorts (Fig. 8) showed that the nomograms were also able to stratify each AJCC7 stage into three significant prognostic groups with low, medium, and high risks of CSS and OS, respectively. Additional elaborations on the 5 -year cumulative survival (Table 8) and hazard ratios (Table 9) of the Nomo stages as well as the stratified risk groups (Table 10) confirmed robust utility of nomograms in both risk classification and stratification.

Finally, the mosaic plots demonstrated significant survival heterogeneity within individual AJCC7 substages in contrast to the Nomo stages (Fig. 9). The results offer direct evidence and the underlying frequencies of staging errors in the conventional AJCC staging system.

\section{Decision curve analysis}

After addressing the model accuracy, DCA was applied to render clinical validity to the nomograms in the derivation cohort and generalize it to the external cohorts. The results corroborated good clinical applicability of the nomograms in predicting survival of patients with $\mathrm{CRC}$ because their ranges of threshold probabilities were wide and practical in all cohorts (Fig. 10). Additional comparisons of model competence were also in favor of the nomograms' superiority over the conventional AJCC stages because the net benefit for the patients was consistently enhanced (higher lines for model prediction relative to the horizontal lines) when using the nomograms compared with using the TNM stages (Fig. 10).

\section{Discussion}

In the present study, we developed two postoperative nomograms to predict CSS and OS for patients who have undergone CRC resection without neoadjuvant therapy. The nomograms consistently achieved considerable predictive accuracy and appreciable reliability and reproducibility when applied to the derivation and validation cohorts. DCA subsequently demonstrated significant clinical applicability of the nomograms with wide threshold probabilities. In addition, model comparisons and DCA proved that the nomograms outperformed the conventional AJCC stages by stratifying them into three significant prognostic groups and allowing for more robust risk classification (Nomo stages) with an improved net benefit.

Prognostic nomograms are simplified representations of complicated statistical models with elegant graphics $[18,29,30]$. Compared with other predictive models, they are more accurate and comprehensible with userfriendly interfaces, allowing for wide application in clinical practice $[18,29,30]$. A recent systematic review summarized the basic characteristics of more than 16

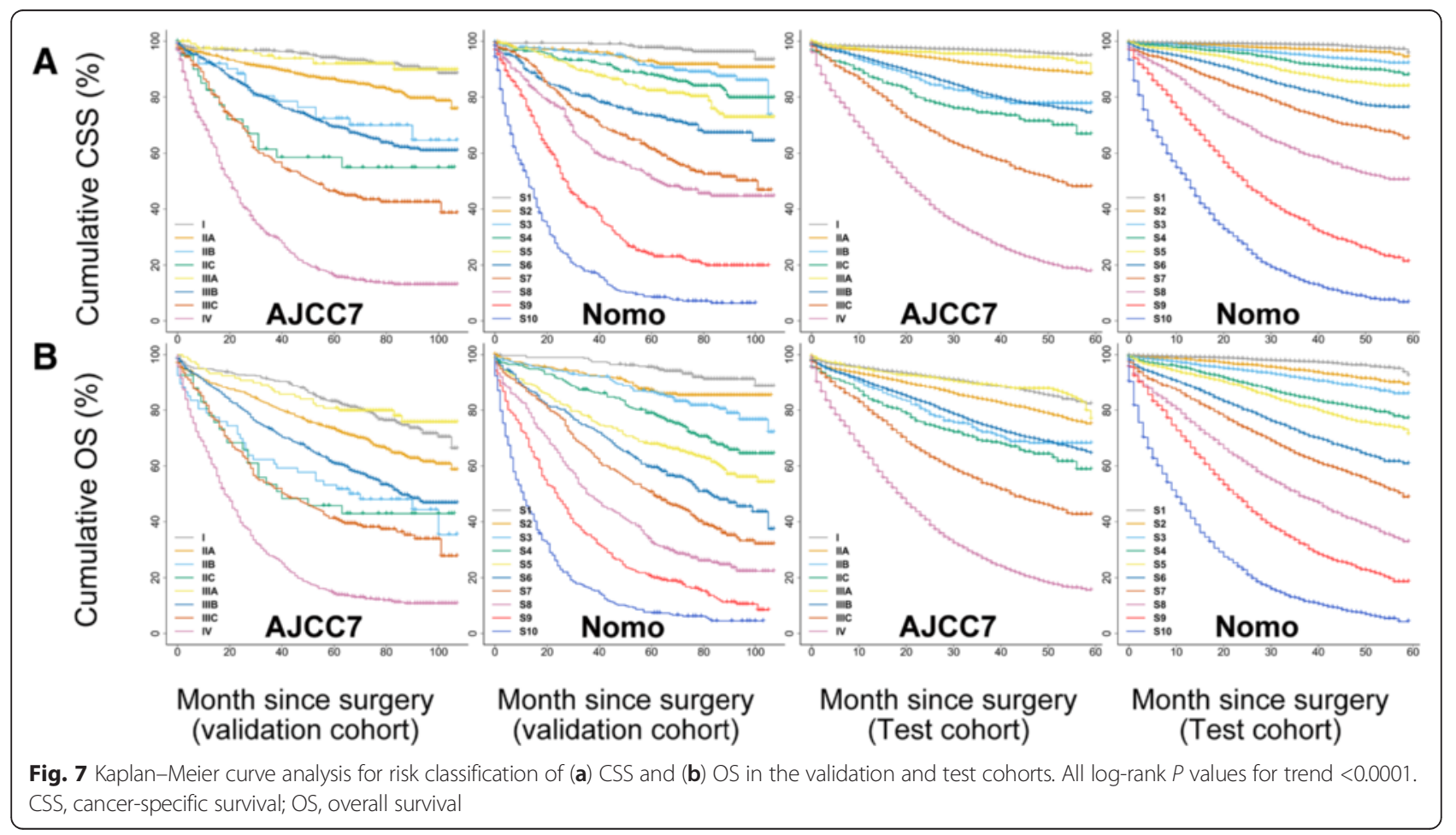


predictive nomograms for CRC [31]. Although patient definitions, endpoints, and time points are markedly heterogeneous, most of the nomograms have demonstrated improved accuracy. Our study shows some distinctions from those published nomograms, however.

First, no previous studies incorporated both patients with non-metastatic CRC and those with metastatic CRC. Because both non-metastatic and metastatic CRC are continuous representations of systemic tumor biology, exclusion of patients with metastasis may inherit the limitations of the AJCC stages. Second, we used population-based data to derive nomograms for CRC; this may be considered an update and extension of a previously published nomogram that also used SEER data but concentrated on curative stage I to III colonic adenocarcinomas [32]. Population-based data often fail to include detailed data and novel markers such as the CEA concentration [19] and microRNAs [33], which may be helpful to increase model accuracy. However, population-based data are more likely to overcome inconsistency biased by institutional practice [18]. Third, we selected covariates based on the AIC instead of statistical significance ( $P$ value), allowing for confidence in the robustness of modeling and performance [34]. The $P$ values depend not only on the magnitude of the predictors' effects but also on the sample size. Small data sets are less likely to discriminate small differences, and their use makes it more difficult to reject the null hypothesis. We also used restricted cubic spline functions for continuous variables to avoid unnecessary information loss caused by categorization [23]. Finally, we introduced DCA and proved the clinical validity of our nomograms. High predictive accuracy is not necessarily associated with usefulness in clinical practice. Wellperforming models may have limited applicability if the threshold probabilities of the net benefits are impractical, meaning that the new predictive models will be less beneficial than currently available tools and may even be harmful $[18,26]$.

Our study also produced some novel findings besides the many results consistent with previous studies. Above all, based on the nomograms, we have proposed Nomo stages and efficiently classified stage I to IV CRCs into 10 significant subgroups with a single predictive score.

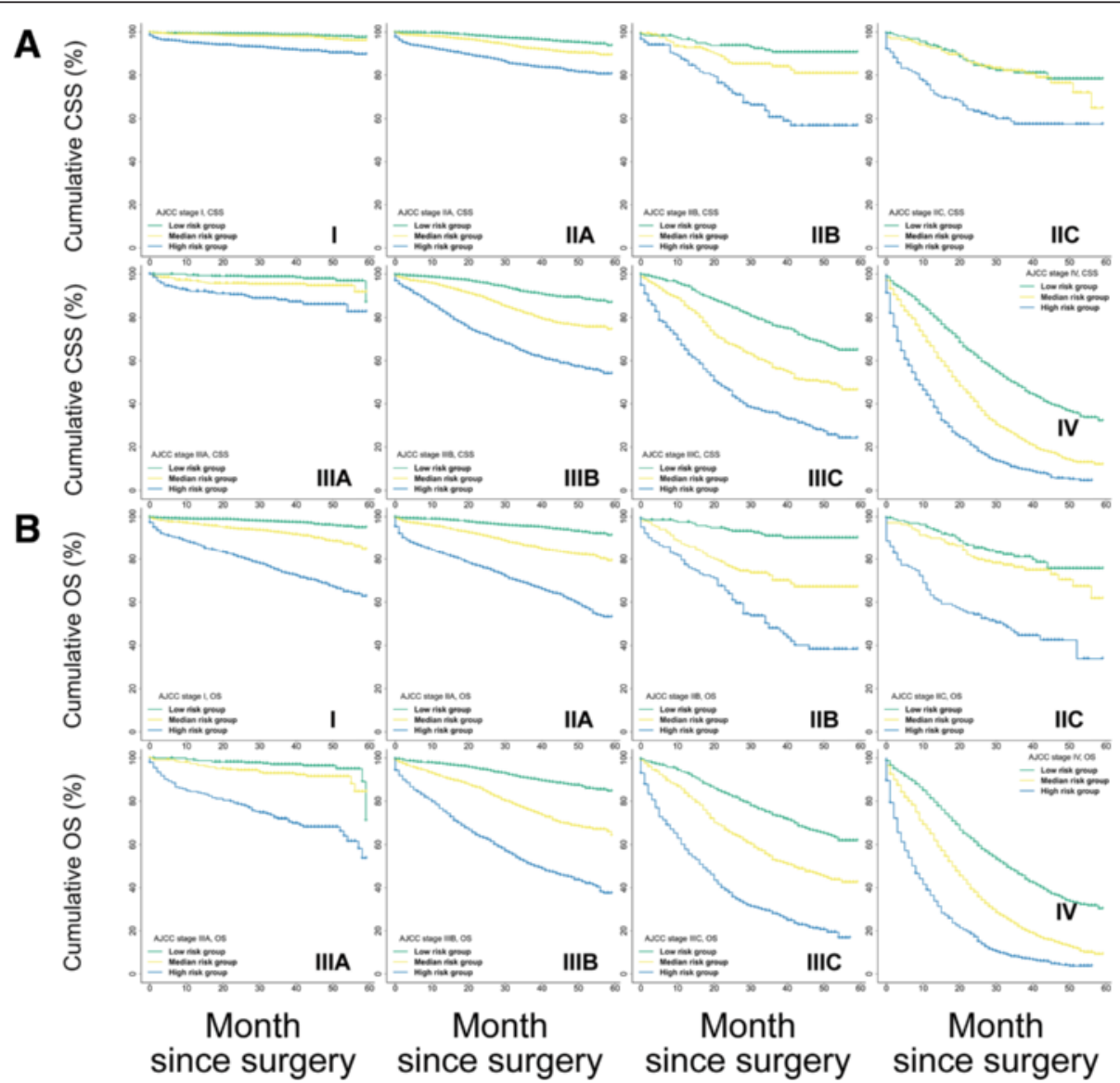

Fig. 8 Kaplan-Meier curve analysis for risk stratification of (a) CSS and (b) OS within each AJCC substage using the test cohort. All log-rank $P$ values for trend $<0.0001$. CSS, cancer-specific survival; OS, overall survival 
Table 8 Cumulative survival for Nomo stages in derivation and external validation cohorts

\begin{tabular}{|c|c|c|c|c|c|c|c|c|c|c|c|c|}
\hline \multirow[b]{2}{*}{ Nomo stage } & \multicolumn{4}{|c|}{$\begin{array}{l}\text { Training cohort } \\
\text { (Cumulative survival, } 60 \text { months, \%) }\end{array}$} & \multicolumn{4}{|c|}{$\begin{array}{l}\text { Validation cohort } \\
\text { (Cumulative survival, } 60 \text { months, \%) }\end{array}$} & \multicolumn{4}{|c|}{$\begin{array}{l}\text { Test cohort } \\
\text { (Cumulative survival, } 59 \text { months, \%) }\end{array}$} \\
\hline & CSS & $95 \% \mathrm{Cl}$ & OS & $95 \% \mathrm{Cl}$ & CSS & $95 \% \mathrm{Cl}$ & OS & $95 \% \mathrm{Cl}$ & CSS & $95 \% \mathrm{Cl}$ & OS & $95 \%$ Cl \\
\hline Nomo 1 & 96.8 & $96.1-97.4$ & 94.6 & $93.7-95.4$ & 97.7 & $96.1-99.4$ & 95.5 & $93.2-97.8$ & 94.3 & $88.7-99.8$ & 90.8 & $85.5-96.1$ \\
\hline Nomo 2 & 94.0 & $93.1-95.0$ & 88.1 & 86.9-89.3 & 92.9 & $90.0-95.8$ & 87.1 & 83.4-90.9 & 94.0 & $91.5-96.6$ & 89.0 & 86.5-91.4 \\
\hline Nomo 3 & 90.9 & $89.8-92.0$ & 83.7 & $82.4-85.1$ & 91.2 & $88.0-94.4$ & 86.6 & $82.8-90.3$ & 92.1 & $90.5-93.6$ & 85.5 & $83.4-87.7$ \\
\hline Nomo 4 & 87.1 & $85.8-88.4$ & 76.1 & $74.5-77.7$ & 88.1 & 84.4-91.7 & 78.9 & $74.4-83.4$ & 87.4 & $84.5-90.3$ & 76.5 & $73.5-79.5$ \\
\hline Nomo 5 & 82.0 & $80.4-83.5$ & 70.3 & $68.6-72.0$ & 82.3 & 77.9-86.7 & 67.8 & $62.6-73.0$ & 83.7 & $81.8-85.7$ & 69.1 & $62.1-76.1$ \\
\hline Nomo 6 & 74.2 & $72.4-75.9$ & 59.0 & $57.2-60.9$ & 73.6 & $68.5-78.7$ & 59.4 & $54.0-64.8$ & 76.1 & 73.9-78.4 & 60.1 & 56.9-63.3 \\
\hline Nomo 7 & 63.3 & $61.4-65.2$ & 44.8 & $42.9-46.7$ & 61.3 & $55.6-67.0$ & 50.6 & $45.1-56.1$ & 64.4 & $60.3-68.6$ & 47.4 & $43.3-51.5$ \\
\hline Nomo 8 & 43.6 & $41.6-45.6$ & 32.6 & $30.9-34.4$ & 50.8 & $44.8-56.8$ & 32.7 & $27.5-37.9$ & 49.9 & $46.9-53.0$ & 31.8 & 27.9-35.7 \\
\hline Nomo 9 & 22.1 & $20.4-23.7$ & 18.0 & $16.5-19.4$ & 23.9 & 18.8-29.0 & 20.2 & $15.8-24.7$ & 20.2 & $16.0-24.4$ & 17.8 & 15.0-20.6 \\
\hline Nomo 10 & 5.7 & $4.7-6.6$ & 4.8 & $4.0-5.6$ & 8.4 & $5.2-11.6$ & 7.3 & $4.5-10.2$ & 6.3 & $3.9-8.6$ & 3.8 & $1.3-6.3$ \\
\hline$P_{\text {log-rank }}$ for trend & $<0.0001$ & & $<0.0001$ & & $<0.0001$ & & $<0.0001$ & & $<0.0001$ & & $<0.0001$ & \\
\hline
\end{tabular}

CSS cancer-specific survival, OS overall survival, Nomo Nomo stages

Table 9 Relative hazard for Nomo stages in derivation and external validation cohorts

\begin{tabular}{|c|c|c|c|c|c|c|c|c|c|c|}
\hline \multirow[b]{2}{*}{ Nomo stages } & \multirow[b]{2}{*}{ Cut-off Points } & \multicolumn{3}{|c|}{ Training cohort } & \multicolumn{3}{|c|}{ Validation cohort } & \multicolumn{3}{|c|}{ Test cohort } \\
\hline & & $\mathrm{HR}$ & $95 \% \mathrm{Cl}$ & $P$ & $\mathrm{HR}$ & $95 \% \mathrm{Cl}$ & $P$ & $\mathrm{HR}$ & $95 \% \mathrm{Cl}$ & $P$ \\
\hline \multicolumn{11}{|c|}{ Cancer-specific survival } \\
\hline Nomo 1 & $\leq 82.0$ & ref & & & ref & & & ref & & \\
\hline Nomo 2 & $\leq 106.2$ & 1.86 & $1.48-2.35$ & $<0.0001$ & 2.40 & $1.19-4.84$ & 0.0147 & 1.92 & $1.37-2.70$ & 0.0002 \\
\hline Nomo 3 & $\leq 122.9$ & 2.86 & $2.30-3.55$ & $<0.0001$ & 3.33 & $1.70-6.51$ & 0.0004 & 3.38 & $2.48-4.62$ & $<0.0001$ \\
\hline Nomo 4 & $\leq 138.2$ & 3.91 & $3.17-4.82$ & $<0.0001$ & 4.83 & $2.54-9.22$ & $<0.0001$ & 5.13 & $3.81-6.91$ & $<0.0001$ \\
\hline Nomo 5 & $\leq 153.0$ & 5.58 & $4.55-6.84$ & $<0.0001$ & 6.60 & $3.50-12.42$ & $<0.0001$ & 7.87 & $5.89-10.51$ & $<0.0001$ \\
\hline Nomo 6 & $\leq 170.7$ & 8.47 & $6.95-10.33$ & $<0.0001$ & 10.41 & $5.60-19.32$ & $<0.0001$ & 12.17 & $9.19-16.13$ & $<0.0001$ \\
\hline Nomo 7 & $\leq 192.2$ & 12.46 & $10.25-15.14$ & $<0.0001$ & 16.23 & $8.84-29.81$ & $<0.0001$ & 18.39 & $13.91-24.3$ & $<0.0001$ \\
\hline Nomo 8 & $\leq 225.6$ & 22.40 & $18.49-27.13$ & $<0.0001$ & 21.11 & $11.53-38.67$ & $<0.0001$ & 32.19 & $24.47-42.34$ & $<0.0001$ \\
\hline Nomo 9 & $\leq 272.1$ & 40.47 & $33.46-48.94$ & $<0.0001$ & 42.87 & $23.56-78.00$ & $<0.0001$ & 65.32 & $49.79-85.68$ & $<0.0001$ \\
\hline Nomo 10 & $272.1+$ & 83.31 & 68.9-100.73 & $<0.0001$ & 86.07 & 47.39-156.31 & $<0.0001$ & 127.46 & $97.2-167.12$ & $<0.0001$ \\
\hline \multicolumn{11}{|l|}{ Overall survival } \\
\hline Nomo 1 & $\leq 57.2$ & ref & & & ref & & & ref & & \\
\hline Nomo 2 & $\leq 70.0$ & 2.16 & $1.83-2.56$ & $<0.0001$ & 1.96 & $1.19-3.22$ & 0.0078 & 2.13 & $1.68-2.70$ & $<0.0001$ \\
\hline Nomo 3 & $\leq 80.8$ & 3.04 & $2.59-3.58$ & $<0.0001$ & 2.63 & $1.64-4.21$ & 0.0001 & 3.12 & $2.49-3.92$ & $<0.0001$ \\
\hline Nomo 4 & $\leq 90.7$ & 4.76 & $4.08-5.56$ & $<0.0001$ & 4.28 & $2.73-6.71$ & $<0.0001$ & 5.55 & $4.48-6.87$ & $<0.0001$ \\
\hline Nomo 5 & $\leq 101.6$ & 6.12 & $5.26-7.12$ & $<0.0001$ & 6.17 & $3.99-9.55$ & $<0.0001$ & 6.90 & $5.59-8.52$ & $<0.0001$ \\
\hline Nomo 6 & $\leq 114.2$ & 9.01 & $7.76-10.45$ & $<0.0001$ & 8.41 & $5.47-12.92$ & $<0.0001$ & 11.11 & $9.06-13.63$ & $<0.0001$ \\
\hline Nomo 7 & $\leq 129.1$ & 13.46 & $11.62-15.58$ & $<0.0001$ & 11.25 & 7.36-17.19 & $<0.0001$ & 15.23 & $12.45-18.62$ & $<0.0001$ \\
\hline Nomo 8 & $\leq 147.1$ & 18.68 & $16.15-21.61$ & $<0.0001$ & 16.81 & $11.04-25.61$ & $<0.0001$ & 24.23 & $19.85-29.57$ & $<0.0001$ \\
\hline Nomo 9 & $\leq 171.8$ & 28.44 & $24.61-32.88$ & $<0.0001$ & 25.47 & $16.76-38.68$ & $<0.0001$ & 37.66 & $30.91-45.89$ & $<0.0001$ \\
\hline Nomo 10 & $171.8+$ & 55.25 & $47.8-63.86$ & $<0.0001$ & 45.27 & $29.80-68.76$ & $<0.0001$ & 75.07 & 61.63-91.45 & $<0.0001$ \\
\hline
\end{tabular}


Table 10 Risk stratifications for each AJCC substage in training and test cohorts

\begin{tabular}{|c|c|c|c|c|c|c|c|c|}
\hline \multirow[b]{2}{*}{ AJCC stages } & \multirow[b]{2}{*}{ Cut-off Points } & \multicolumn{4}{|l|}{ Training cohort } & \multicolumn{3}{|c|}{ Test cohort } \\
\hline & & $\begin{array}{l}\text { Cumulative Survival, } \\
60 \text { months, \% }\end{array}$ & $\mathrm{HR}$ & $95 \% \mathrm{Cl}$ & Pairwise $P_{\text {log-rank }}$ & $\mathrm{HR}$ & $95 \% \mathrm{Cl}$ & Pairwise $P_{\text {log-rank }}$ \\
\hline \multicolumn{9}{|l|}{ Cancer-specific survival } \\
\hline \multicolumn{9}{|l|}{ Stage I } \\
\hline Low risk group $(\mathrm{L})$ & $\leq 70.0$ & 97.7 & ref & & $L \vee M<0.0001$ & ref & & $L \vee M<0.0205$ \\
\hline Median risk group (M) & $\leq 97.2$ & 94.4 & 2.44 & $1.91-3.13$ & $L \vee H<0.0001$ & 1.75 & $1.24-2.45$ & $L \vee H<0.0001$ \\
\hline High risk group $(H)$ & $97.2+$ & 88.4 & 4.73 & $3.66-6.12$ & $\mathrm{M} \vee \mathrm{H}<0.0001$ & 6.12 & $4.27-8.77$ & $M \vee H<0.0001$ \\
\hline \multicolumn{9}{|l|}{ Stage IIA } \\
\hline Low risk group (L) & $\leq 122.7$ & 93.9 & ref & & $L \vee M<0.0001$ & ref & & $L \vee M<0.0001$ \\
\hline Median risk group (M) & $\leq 149.4$ & 87.7 & 1.90 & $1.64-2.19$ & $L \vee H<0.0001$ & 2.11 & $1.4-2.58$ & $L \vee H<0.0001$ \\
\hline High risk group $(\mathrm{H})$ & $149.4+$ & 76.2 & 3.96 & $3.41-4.61$ & $M \vee H<0.0001$ & 4.66 & $3.80-5.72$ & $M \vee H<0.0001$ \\
\hline \multicolumn{9}{|l|}{ Stage IIB } \\
\hline Low risk group $(\mathrm{L})$ & $\leq 151.2$ & 84.4 & ref & & $L \vee M=0.0046$ & ref & & $L \vee M=0.0220$ \\
\hline Median risk group (M) & $\leq 178.4$ & 71.1 & 1.84 & $1.28-2.64$ & $L \vee H<0.0001$ & 2.09 & $1.29-3.39$ & $L \vee H<0.0001$ \\
\hline High risk group $(H)$ & $178.4+$ & 54.1 & 3.30 & $2.22-4.90$ & $\mathrm{M} \vee \mathrm{H}<0.0012$ & 5.27 & $3.16-8.81$ & $M \vee H<0.0001$ \\
\hline \multicolumn{9}{|l|}{ Stage IIC } \\
\hline Low risk group (L) & $\leq 158.0$ & 76.7 & ref & & $L \vee M=0.0121$ & ref & & $L \vee M=0.6571$ \\
\hline Median risk group (M) & $\leq 181.8$ & 62.3 & 1.64 & $1.16-2.31$ & $\mathrm{~L} \vee \mathrm{H}<0.0001$ & 1.12 & $0.72-1.74$ & $L \vee H<0.0001$ \\
\hline High risk group $(H)$ & $181.8+$ & 45.6 & 2.79 & $1.93-2.03$ & $\mathrm{M} \vee \mathrm{H}<0.0020$ & 2.73 & $1.68-4.42$ & $M \vee H<0.0001$ \\
\hline \multicolumn{9}{|l|}{ Stage IIIA } \\
\hline Low risk group (L) & $\leq 88.9$ & 94.8 & ref & & $L \vee M=0.0116$ & ref & & $L \vee M<0.0210$ \\
\hline Median risk group (M) & $\leq 120.2$ & 91.6 & 2.04 & $1.39-2.99$ & $L \vee H<0.0001$ & 2.51 & $1.36-4.63$ & $L \vee H<0.0001$ \\
\hline High risk group $(H)$ & $120.2+$ & 75.6 & 6.84 & $4.57-10.24$ & $\mathrm{M} \vee \mathrm{H}<0.0001$ & 6.54 & $3.20-13.35$ & $M \vee H=0.0015$ \\
\hline \multicolumn{9}{|l|}{ Stage IIIB } \\
\hline Low risk group (L) & $\leq 147.6$ & 85.9 & ref & & $L \vee M<0.0001$ & ref & & $L \vee M<0.0001$ \\
\hline Median risk group (M) & $\leq 180.3$ & 72.1 & 2.09 & $1.89-2.32$ & $L \vee H<0.0001$ & 2.35 & $2.04-2.70$ & $L \vee H<0.0001$ \\
\hline High risk group $(\mathrm{H})$ & $180.3+$ & 50.1 & 4.25 & $3.80-4.76$ & $\mathrm{M} \vee \mathrm{H}<0.0001$ & 5.34 & $4.55-2.67$ & $M \vee H<0.0001$ \\
\hline \multicolumn{9}{|l|}{ Stage IIIC } \\
\hline Low risk group (L) & $\leq 187.2$ & 65.1 & ref & & $L \vee M<0.0001$ & ref & & $L \vee M<0.0001$ \\
\hline Median risk group (M) & $\leq 218.0$ & 41.6 & 1.99 & $1.74-2.27$ & $L \vee H<0.0001$ & 2.01 & $1.72-2.36$ & $L \vee H<0.0001$ \\
\hline High risk group $(H)$ & $218.0+$ & 24.8 & 3.49 & $3.01-4.04$ & $\mathrm{M} \vee \mathrm{H}<0.0001$ & 4.05 & $3.39-4.84$ & $M \vee H<0.0001$ \\
\hline \multicolumn{9}{|l|}{ Stage IV } \\
\hline Low risk group (L) & $\leq 255.6$ & 26.2 & ref & & $L \vee M<0.0001$ & ref & & $L \vee M<0.0001$ \\
\hline Median risk group (M) & $\leq 292.1$ & 12.0 & 1.56 & $1.46-1.67$ & $\mathrm{~L} \vee \mathrm{H}<0.0001$ & 1.85 & $1.70-2.01$ & $L \vee H<0.0001$ \\
\hline High risk group $(H)$ & $292.1+$ & 4.0 & 2.78 & $2.57-3.01$ & $\mathrm{M} \vee \mathrm{H}<0.0001$ & 3.34 & $3.20-3.70$ & $M \vee H<0.0001$ \\
\hline \multicolumn{9}{|l|}{ Overall survival } \\
\hline \multicolumn{9}{|l|}{ Stage I } \\
\hline Low risk group (L) & $\leq 55.1$ & 94.6 & ref & & $L \vee M<0.0001$ & ref & & $L \vee M<0.0001$ \\
\hline Median risk group (M) & $\leq 79.9$ & 84.5 & 2.94 & $2.58-3.35$ & $L \vee H<0.0001$ & 3.10 & $2.58-3.72$ & $L \vee H<0.0001$ \\
\hline High risk group $(\mathrm{H})$ & $79.9+$ & 63.1 & 9.07 & $7.90-10.41$ & $\mathrm{M} \vee \mathrm{H}<0.0001$ & 10.70 & $8.80-13.01$ & $\mathrm{M} \vee \mathrm{H}<0.0001$ \\
\hline \multicolumn{9}{|l|}{ Stage IIA } \\
\hline Low risk group (L) & $\leq 75.9$ & 90.6 & ref & & $L \vee M<0.0001$ & ref & & $L \vee M<0.0001$ \\
\hline Median risk group (M) & $\leq 101.4$ & 77.0 & 2.66 & $2.42-2.91$ & $L \vee H<0.0001$ & 2.86 & $2.50-3.28$ & $L \vee H<0.0001$ \\
\hline High risk group $(\mathrm{H})$ & $101.4+$ & 49.1 & 7.38 & $6.68-8.15$ & $M \vee H<0.0001$ & 7.80 & $6.77-8.97$ & $M \vee H<0.0001$ \\
\hline
\end{tabular}


Table 10 Risk stratifications for each AJCC substage in training and test cohorts (Continued)

\begin{tabular}{|c|c|c|c|c|c|c|c|c|}
\hline \multicolumn{9}{|l|}{ Stage IIB } \\
\hline Low risk group (L) & $\leq 93.5$ & 80.3 & ref & & $L \vee M=0.0007$ & ref & & $L \vee M<0.0001$ \\
\hline Median risk group (M) & $\leq 120.4$ & 64.7 & 1.86 & $1.40-2.45$ & $L \vee H<0.0001$ & 3.86 & $2.60-5.72$ & $L \vee H<0.0001$ \\
\hline High risk group $(H)$ & $120.4+$ & 26.8 & 5.48 & $4.00-7.49$ & $\mathrm{M} \vee \mathrm{H}<0.0001$ & 8.03 & $5.27-12.22$ & $M \vee H<0.0001$ \\
\hline \multicolumn{9}{|l|}{ Stage IIC } \\
\hline Low risk group (L) & $\leq 96.9$ & 73.6 & ref & & $L \vee M<0.0001$ & ref & & $L \vee M=0.2111$ \\
\hline Median risk group (M) & $\leq 121.9$ & 54.4 & 2.09 & $1.56-2.80$ & $L \vee H<0.0001$ & 1.35 & $0.92-1.98$ & $L \vee H<0.0001$ \\
\hline High risk group $(H)$ & $121.9+$ & 35.5 & 3.54 & $2.58-4.86$ & $\mathrm{M} \vee \mathrm{H}=0.0002$ & 3.81 & $2.46-5.91$ & $M \vee H<0.0001$ \\
\hline \multicolumn{9}{|l|}{ Stage IIIA } \\
\hline Low risk group (L) & $\leq 60.0$ & 94.6 & ref & & $L \vee M<0.0001$ & ref & & $L \vee M<0.0316$ \\
\hline Median risk group (M) & $\leq 90.4$ & 84.3 & 3.67 & $2.76-4.87$ & $L \vee H<0.0001$ & 2.10 & $1.36-3.25$ & $L \vee H<0.0001$ \\
\hline High risk group $(H)$ & $90.4+$ & 59.4 & 11.71 & $8.66-15.84$ & $\mathrm{M} \vee \mathrm{H}<0.0001$ & 9.07 & $5.70-14.45$ & $M \vee H<0.0001$ \\
\hline \multicolumn{9}{|l|}{ Stage IIIB } \\
\hline Low risk group (L) & $\leq 91.0$ & 82.3 & ref & & $L \vee M<0.0001$ & ref & & $L \vee M<0.0001$ \\
\hline Median risk group (M) & $\leq 116.9$ & 63.7 & 2.28 & $2.09-2.48$ & $\mathrm{~L} \vee \mathrm{H}<0.0001$ & 2.72 & $2.42-3.07$ & $L \vee H<0.0001$ \\
\hline High risk group $(\mathrm{H})$ & $116.9+$ & 36.6 & 5.06 & $4.60-5.56$ & $\mathrm{M} \vee \mathrm{H}<0.0001$ & 6.55 & $5.75-7.47$ & $M \vee H<0.0001$ \\
\hline \multicolumn{9}{|l|}{ Stage IIIC } \\
\hline Low risk group (L) & $\leq 110.6$ & 63.3 & ref & & $L \vee M<0.0001$ & ref & & $L \vee M<0.0001$ \\
\hline Median risk group (M) & $\leq 137.0$ & 35.7 & 2.10 & $1.86-2.37$ & $L \vee H<0.0001$ & 1.94 & $1.68-2.24$ & $L \vee H<0.0001$ \\
\hline High risk group $(H)$ & $137.0+$ & 18.2 & 3.97 & $3.47-4.55$ & $M \vee H<0.0001$ & 4.33 & $3.68-5.11$ & $M \vee H<0.0001$ \\
\hline \multicolumn{9}{|l|}{ Stage IV } \\
\hline Low risk group (L) & $\leq 157.2$ & 24.2 & ref & & $L \vee M<0.0001$ & ref & & $L \vee M<0.0001$ \\
\hline Median risk group (M) & $\leq 183.3$ & 9.9 & 1.59 & $1.49-1.69$ & $L \vee H<0.0001$ & 1.87 & $1.73-2.03$ & $\mathrm{~L} \vee \mathrm{H}<0.0001$ \\
\hline High risk group $(\mathrm{H})$ & $183.3+$ & 3.0 & 2.79 & $2.58-3.01$ & $M \vee H<0.0001$ & 3.51 & $3.19-3.88$ & $M \vee H<0.0001$ \\
\hline
\end{tabular}

AJCC American joint committee on cancer, $H R$ hazard ratio, $95 \% \mathrm{Cl} 95 \%$ confident interval, $L$ low risk group, $M$ median risk group, $H$ high risk group

Our nomograms also enable stratification of each AJCC7 substage into three significant risk strata, which has not been achieved by other CRC nomograms. This risk classification and stratification may be very useful for clinicians to identify postoperative patients with high risks associated with intensified follow-up (i.e., patients with high-risk stage I CRC) and select less heterogeneous patients for clinical trials (i.e., patients with high-risk stage II CRC). This also helps to understand the degree of survival heterogeneity in the AJCC stages, which frequently introduces confusion and uncertainty to patient consulting. Note that the optimal thresholds for risk classification and stratification may be individualized, although the thresholds defined by the training cohort still worked well in our external cohorts, which are only intended for relatively strict validation. Additionally and importantly, the sharing of similar contributing predictors is a reflection of apparent correlations between CSS and OS. Some of these predictors are worth noting here. In our models, age had a persistent effect but multiplied from beyond 60 years old. Age is a traditional reference for physical condition, frequency and efficiency of reinforced therapies, thus exerts an accumulated effect on survival. It is reasonable to presume that certain tumor-related factors such as infiltration depth, metastasis, histology, LNR, and LNC are relatively more important predictors than age. They are typical features of tumor development and are closely related to patient death at various but statistically significant levels. The LNC is one of the most controversial among these tumor-related factors. It has been proposed as a quality indicator $[6,35]$ and is augmented in extended lymphadenectomy, the relevant long-term benefit of which has not been effectively demonstrated because of the absence of prospective clinical trials of extended colonic surgeries [35, 36]. Inadequate LNC assessment is involved in interpretation of stage migration, which is considered a source of survival heterogeneity in patients with CRC, but its influences are limited [14, 35, 36]. Several previous studies classified patients by the 12-node benchmark to derive high- and low-risk subpopulations but achieved inconsistent results, while our results indicate that such classification might be associated with a risk of dichotomizing complex, non-linear effects of LNC on patient survival [37]. Moreover, our analyses indicated that LNC was less superior to LNR, which 


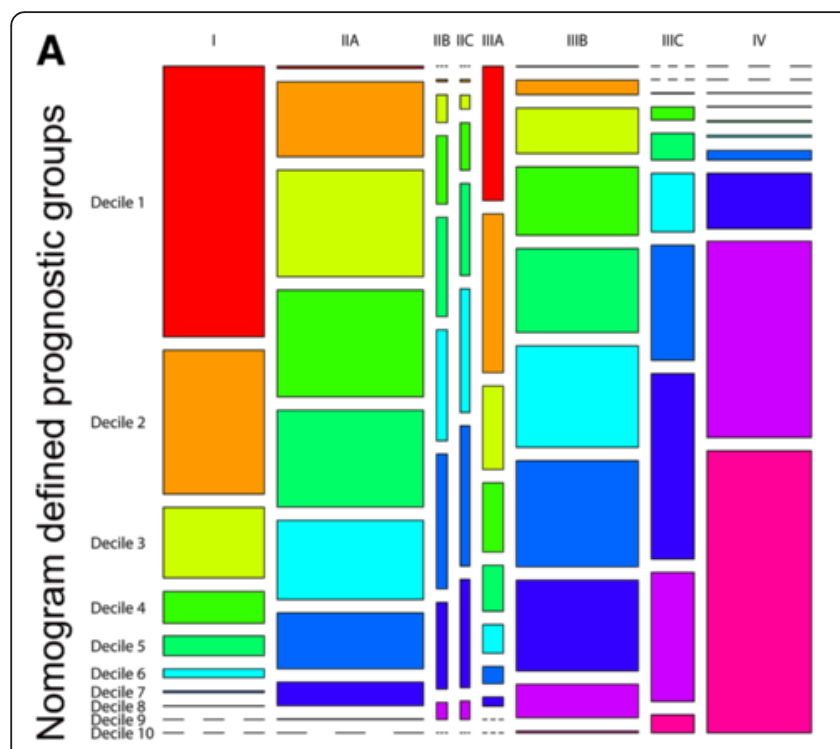

7th AJCC stages

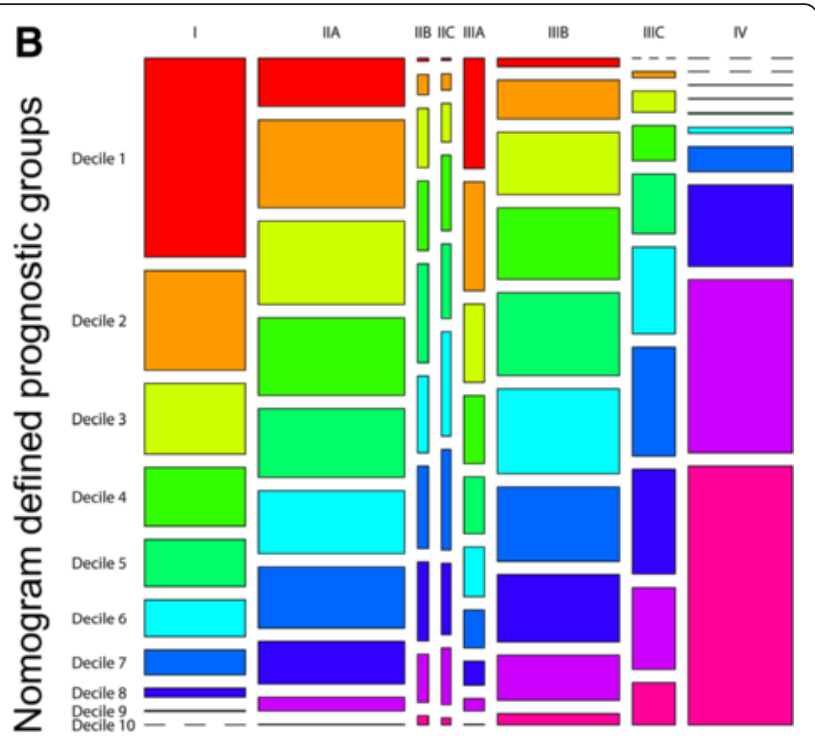

7th AJCC stages

Fig. 9 Mosaic plots using the training cohort. a CSS, b OS. In the mosaic plots, each of the 10 deciles is represented by 1 of 10 consecutive rainbow colors. The area of the individual mosaics represents the relative frequency associated with the column cell. The short segmented lines indicate a frequency of zero. CSS, cancer-specific survival; OS, overall survival; AJCC, American Joint Committee on Cancer

explains the reduced survival in the patient subset with limited numbers of metastatic nodes. Additionally, the preoperative CEA concentration provides a baseline quantification of the tumor burden and severity of disease. The CEA concentration, with its individualized information and wide application, is due to play a role in the staging of CRC. Next, the effects of racial background may be multifactorial. The lowest prevalence and mortality of CRC are seen in East Asians because of the low prevalence of risk factors such as smoking and obesity in this population $[1,2]$. The highest incidence and mortality are seen in black people [2]; this can be ascribed to the lower income, later diagnosis, and less access to high-quality health care in this population [2, 20]. Additionally, marriage makes a prognostic difference [20] and deserves more attention because it may provide compensative mechanisms for improvements in survival. Marital status and ethnicity were introduced to prognostic nomograms for CRC for the first time in the present study. It should also be noted that the nomogram points translated from the models' coefficients reflect the importance of the variables relative to the presence of the other covariates. They may vary depending on the outcomes measured. Due to the existence of competing risks, the predictive accuracy for OS tended to be lower than that for CSS in our study. However, we chose a Cox proportional hazard model without competing risks because it was easier to interpret, compare, and comprehend [38].
Our study has limitations that deserve attention. Improved model accuracy frequently comes at the cost of increased complexity. The tradeoffs between comprehensiveness and comprehensibility are not easy to balance, and this is a common problem during modeling for nomograms. Considering this, we only selected variables that were clinically important and practical with high reproducibility and low time-varying effects. The nomogram itself is associated with uncertainty. Therefore, we provided $95 \%$ CIs for the c-indexes and calibrations to determine the degree of uncertainty. Because of the shorter follow-up, the c-indexes were slightly higher in the test cohort than in the derivation cohort. However, the time-dependent ROC showed that the predictive AUCs of the nomograms in different cohorts were very close in the same years. Moreover, our nomograms were developed for risk assessment and selection of patients who might benefit from additional interventions after surgery. These interventions may include but are not restricted to adjuvant therapies, strengthened treatments, intensified follow-ups, and motivated patient consulting. Even so, nomograms cannot substitute for clinicians' judgments or act as exclusive evidence for clinical decision-making. Finally, details regarding tumor deposit, curability of stage IV CRC, and postoperative chemoradiotherapy among the patients in the present study are unknown, placing a limitation on the survival analysis. Incorporation of the new predictors and introduction of competing risk models may further improve 


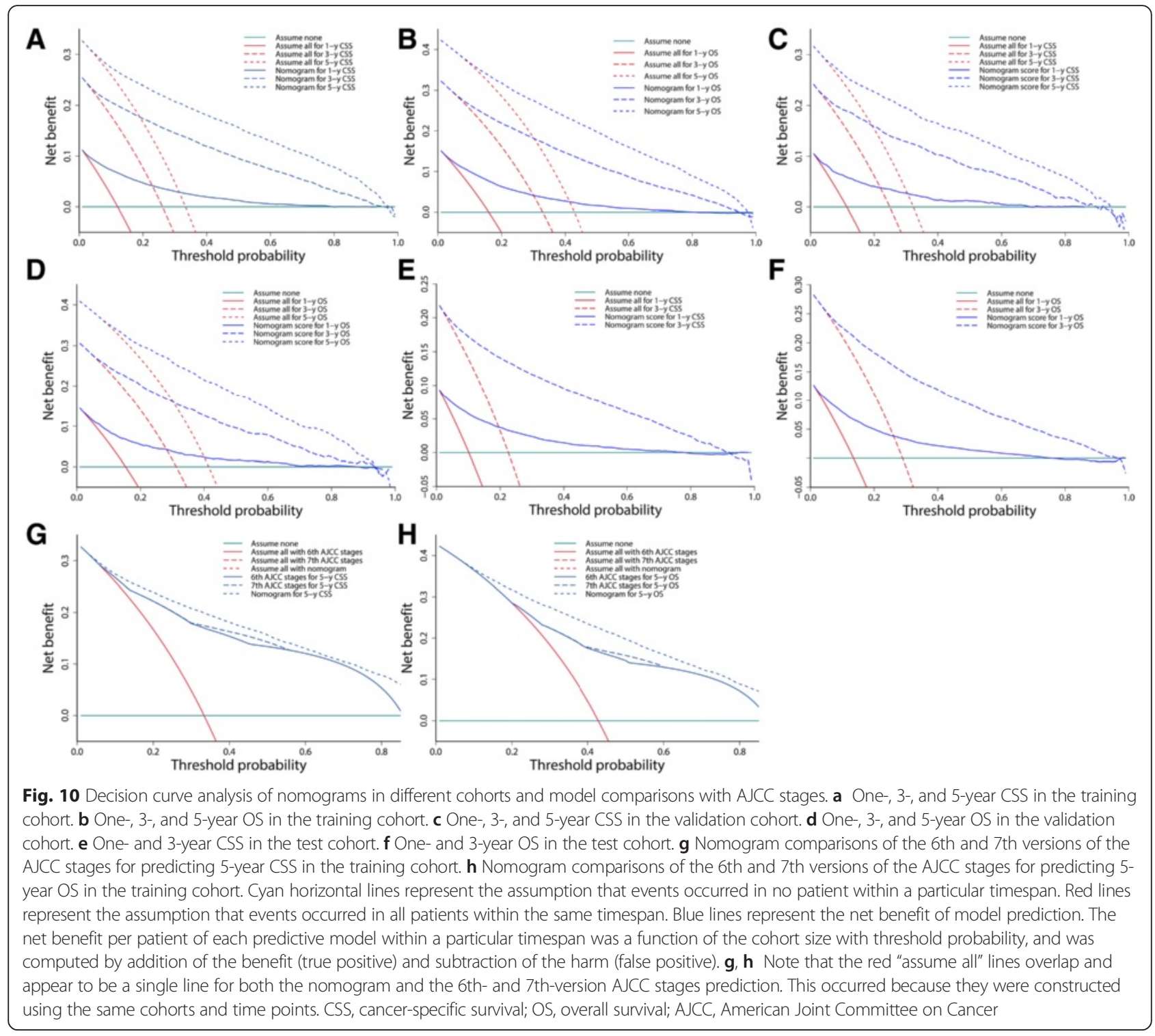

model performance $[18,29]$. However, this will require new nomograms with different modeling strategies.

\section{Conclusions}

In the present study, the bootstrap-corrected and prospectively validated nomograms were consistently reliable and clinically practical with wide threshold probabilities. Moreover, the nomograms outperformed the conventional AJCC stages by allowing for more robust risk classification and stratifying the AJCC stages with an improved net benefit. However, independent external validations are still required in the future.

\section{Acknowledgements}

We thank Dr Cathy Chen and Dr Anthony Lodge for their suggestions with manuscript editing.

\section{Funding}

This study was supported by grants from the Shanghai Science Committee Foundation (no. 34119b0600; no. 16411970800), Shanghai Municipal Health Bureau (no. 20134194), Jiaxing Science Committee Foundation of Zhejiang province (no. 2015AY23071) and the Technology Plan Project of Medicine and Health in Zhejiang Province (no. 2016KYB295).

\section{Availability of data and materials}

The cohort data are available to researchers and should be requested under the approval of the SEER Program administration. The other datasets supporting the conclusions of this article are included within the article.

\section{Authors' contributions}

Study conception and design: HYG; acquisition of data: ZYZ, QFL, XWY, ZLD, SB, HYG; analyses and interpretation of data: ZYZ, QFL, XWY, ZLD, SB, HYG; all authors have read and approved the final version of the manuscript.

\section{Competing interests}

The authors have that they have no competing interests.

Consent for publication

Not applicable. 


\section{Ethics approval and consent to participate}

Because the patients in the SEER database could not be identified, the analysis and reporting of the data in our study were exempt from review by the Ethics Board of Shanghai East Hospital. The requirement for written informed consent to participate was waived. We were permitted to have Internet access after our signed data-use agreement (http://seer.cancer.gov/ data/sample-dua.html) was approved by the SEER administration.

\section{Author details}

${ }^{1}$ Department of Gastrointestinal Surgery, Shanghai East Hospital, Tongji University School of Medicine, Pudong New District, No. 150, Jimo Road, Shanghai 200120, China. ${ }^{2}$ Department of General Surgery, Qingpu Branch of Zhongshan Hospital, Fudan University, Shanghai, China.

\section{Received: 20 May 2016 Accepted: 6 August 2016}

\section{Published online: 19 August 2016}

\section{References}

1. Torre LA, Bray F, Siegel RL, Ferlay J, Lortet-Tieulent J, Jemal A. Global cancer statistics, 2012. CA Cancer J Clin. 2015;65:87-108.

2. Siegel RL, Miller KD, Jemal A. Cancer statistics, 2016. CA Cancer J Clin. 2016; 66:7-30

3. Brenner H, Kloor M, Pox CP. Colorectal cancer. Lancet. 2014;383:1490-502.

4. Schmoll HJ, Van Cutsem E, Stein A, Valentini V, Glimelius B, Haustermans K, et al. ESMO Consensus Guidelines for management of patients with colon and rectal cancer. a personalized approach to clinical decision making. Ann Oncol. 2012;23:2479-516.

5. National Comprehensive Cancer Network. NCCN Clinical Practice Guidelines in Oncology: Colon Cancer. V.2.2016, 2016. https://www.nccn.org/ professionals/physician gls/pdf/colon.pdf. Accessed 31 May 2016.

6. Nagtegaal ID. Current concepts of colorectal cancer resection pathology. Histopathology. 2015;66:102-11.

7. Edge SB, Compton CC. The American Joint Committee on Cancer: the 7th edition of the AJCC cancer staging manual and the future of TNM. Ann Surg Oncol. 2010;17:1471-4.

8. Hyslop T, Weinberg DS, Schulz S, Barkun A, Waldman SA. Occult tumor burden predicts disease recurrence in lymph node-negative colorectal cancer. Clin Cancer Res. 2011;17:3293-303.

9. Benson 3rd AB, Hamilton SR. Path toward prognostication and prediction: an evolving matrix. J Clin Oncol. 2011;29:4599-601.

10. O'Connor ES, Greenblatt DY, LoConte NK, Gangnon RE, Liou Jl, Heise CP, et al. Adjuvant chemotherapy for stage II colon cancer with poor prognostic features. J Clin Oncol. 2011;29:3381-8.

11. Andre T, Boni C, Navarro M, Tabernero J, Hickish T, Topham C, et al. Improved overall survival with Oxaliplatin, fluorouracil, and leucovorin as adjuvant treatment in stage II or III colon cancer in the MOSAIC trial. J Clin Oncol. 2009;27:3109-16.

12. Tougeron D, Mouillet G, Trouilloud I, Lecomte T, Coriat R, Aparicio T, et al. Efficacy of Adjuvant Chemotherapy in Colon Cancer With Microsatellite Instability: A Large Multicenter AGEO Study. J Natl Cancer Inst. 2016;108(7): doi:10.1093/jnci/djv438

13. O'Connell JB, Maggard MA, Ko CY. Colon cancer survival rates with the new American Joint Committee on Cancer sixth edition staging. J Natl Cancer Inst. 2004;96:1420-5

14. Hari DM, Leung AM, Lee JH, Sim MS, Vuong B, Chiu CG, et al. AJCC Cancer Staging Manual 7th edition criteria for colon cancer: do the complex modifications improve prognostic assessment? J Am Coll Surg. 2013;217: $181-90$

15. Kawai K, Ishihara S, Yamaguchi H, Sunami E, Kitayama J, Miyata $H$, et al. Nomograms for predicting the prognosis of stage IV colorectal cancer after curative resection: a multicenter retrospective study. Eur J Surg Oncol. 2015: 41:457-65.

16. Beppu T, Sakamoto Y, Hasegawa K, Honda G, Tanaka K, Kotera Y, et al. A nomogram predicting disease-free survival in patients with colorectal liver metastases treated with hepatic resection: multicenter data collection as a Project Study for Hepatic Surgery of the Japanese Society of Hepato-BiliaryPancreatic Surgery. J Hepatobiliary Pancreat Sci. 2012;19:72-84.

17. Franko J, Shi Q, Goldman CD, Pockaj BA, Nelson GD, Goldberg RM, et al. Treatment of colorectal peritoneal carcinomatosis with systemic chemotherapy: a pooled analysis of north central cancer treatment group phase III trials N9741 and N9841. J Clin Oncol. 2012;30:263-7.
18. Balachandran VP, Gonen M, Smith JJ, DeMatteo RP. Nomograms in oncology: more than meets the eye. Lancet Oncol. 2015;16:e173-180.

19. Weiser MR, Landmann RG, Kattan MW, Gonen M, Shia J, Chou J, et al. Individualized prediction of colon cancer recurrence using a nomogram. J Clin Oncol. 2008;26:380-5.

20. Amri R, Stronks K, Bordeianou LG, Sylla P, Berger DL. Gender and ethnic disparities in colon cancer presentation and outcomes in a US universal health care setting. J Surg Oncol. 2014;109:645-51.

21. Wang L, Wilson SE, Stewart DB, Hollenbeak CS. Marital status and colon cancer outcomes in US Surveillance, Epidemiology and End Results registries: does marriage affect cancer survival by gender and stage? Cancer Epidemiol. 2011;35:417-22.

22. Surveillance, Epidemiology, and End Results (SEER) Program SEER*Stat Database: Incidence - SEER 9 Regs Research Data, Nov 2014 Sub (1973-2012) $<$ Katrina/Rita Population Adjustment > - Linked To County Attributes - Total U.S., 1969-2013 Counties, National Cancer Institute, DCCPS, Surveillance Research Program, Surveillance Systems Branch, released April 2015, based on the November 2014 submission. http://www.seer.cancer.gov. Accessed 31 May 2016.

23. Harrell F. Regression modeling strategies: with applications to linear models, logistic and ordinal regression, and survival analysis. Springer; 2015. http:// www.springer.com/gb/book/9783319194240. Accessed 31 May 2016.

24. Kang L, Chen W, Petrick NA, Gallas BD. Comparing two correlated C indices with right-censored survival outcome: a one-shot nonparametric approach. Stat Med. 2015;34:685-703.

25. Hung $\mathrm{H}$, Chiang $\mathrm{CT}$. Estimation methods for time-dependent AUC models with survival data. Can J Stat. 2010;38:8-26.

26. Vickers AJ, Elkin EB. Decision curve analysis: a novel method for evaluating prediction models. Med Decis Making. 2006;26:565-74.

27. Collins GS, Reitsma JB, Altman DG, Moons KGM. Transparent Reporting of a multivariable prediction model for Individual Prognosis Or Diagnosis (TRIPOD): The TRIPOD Statement. Ann Intern Med. 2015;162:55-63.

28. WMA Declaration of Helsinki. Ethical Principles for Medical Research Involving Human Subjects. http://www.wma.net/en/30publications/ 10policies/b3/index.html. Accessed 31 May 2016.

29. lasonos A, Schrag D, Raj GV, Panageas KS. How to build and interpret a nomogram for cancer prognosis. J Clin Oncol. 2008;26:1364-70.

30. Shariat SF, Karakiewicz PI, Suardi N, Kattan MW. Comparison of nomograms with other methods for predicting outcomes in prostate cancer: A critical analysis of the literature. Clin Cancer Res. 2008;14:4400-7.

31. Kawai K, Sunami E, Yamaguchi H, Ishihara S, Kazama S, Nozawa H, et al. Nomograms for colorectal cancer: a systematic review. World J Gastroenterol. 2015;21:11877-86,

32. Weiser MR, Gonen M, Chou JF, Kattan MW, Schrag D. Predicting survival after curative colectomy for cancer: individualizing colon cancer staging. J Clin Oncol. 2011;29:4796-802

33. Zhang JX, Song W, Chen ZH, Wei JH, Liao YJ, Lei J, et al. Prognostic and predictive value of a microRNA signature in stage II colon cancer: a microRNA expression analysis. Lancet Oncol. 2013;14:1295-306.

34. Pietrantonio F, Aprile G, Rimassa L, Franco P, Lonardi S, Cremolini C, et al. A new nomogram for estimating survival in patients with brain metastases secondary to colorectal cancer. Radiother Oncol. 2015;117:315-21.

35. Wong JH, Lum SS, Morgan JW. Lymph node counts as an indicator of quality at the hospital level in colorectal surgery. J Am Coll Surg. 2011;213:226-30.

36. Willaert W, Mareel M, Van De Putte D, Van Nieuwenhove Y, Pattyn P, Ceelen W. Lymphatic spread, nodal count and the extent of lymphadenectomy in cancer of the colon. Cancer Treat Rev. 2014:40:405-13.

37. Hashiguchi Y, Hase K, Ueno H, Mochizuki H, Shinto E, Yamamoto J. Optimal margins and lymphadenectomy in colonic cancer surgery. Brit I Surg. 2011; 98:1171-8.

38. Valentini V, van Stiphout RGPM, Lammering G, Gambacorta MA, Barba MC Bebenek $M$, et al. Nomograms for predicting local recurrence, distant metastases, and overall survival for patients with locally advanced rectal cancer on the basis of european randomized clinical trials. J Clin Oncol. 2011:29:3163-72. 